\title{
Mandelbrot Market-Model and Momentum
}

\author{
Wilhelm Berghorn ${ }^{1} \&$ Sascha Otto $^{2}$ \\ ${ }^{1}$ Mandelbrot Quantitative Research UG, Helmut-Lederer-Straße 19, 91056 Erlangen, Germany \\ 2 Achterdiek 53b, 28359 Bremen, Germany
}

Correspondence: Dr. Wilhelm Berghorn, Mandelbrot Quantitative Research UG, Helmut-Lederer-Straße 19, 91056

Erlangen, Germany. Tel: 49-9131-930-3725.

Received: April 28, 2017

Accepted: May 23, 2017

Online Published: June 10, 2017

doi:10.5430/ijfr.v8n3p1

URL: https://doi.org/10.5430/ijfr.v8n3p1

\begin{abstract}
Mandelbrot was one of the first who criticized the oversimplifications in finance modeling. In his view, markets have long-term memory, were fractal and thus much wilder than classical theory suggests. Recently, we were able to show that the scaling behaviour of trends, as defined by a specific trend decomposition using wavelets, are causing the momentum effect. In this work, we will show that this effect can be modeled by fractal trends. The so-called Mandelbrot Market-Model shows that markets are wilder compared with classical models. In conclusion, we derive what Mandelbrot always knew: There are no efficient markets.
\end{abstract}

Keywords: efficient market hypothesis, momentum effect, fractal markets, trends

Benoit Mandelbrot is considered by some as the father of quantitative finance as described by Dempster (2011). Among his extraordinary contributions in different disciplines (see Peitgen (2010) for a summary), he has contributed to the field of finance in many different areas. First, he was among the first who criticised normal distribution's usage to model asset returns as originally proposed by Bachelier (1900). Second, Mandelbrot (1963) observed that asset returns exhibit scaling properties, which may indicate that distributions of asset returns have infinite variance. Third, he was able to model "trending" and "mean reversion" effects by introducing, with van Ness in Mandelbrot (1968), the so-called fractional Brownian motion. Finally, Mandelbrot (1997) proposed a combined model using fractional Brownian motion, which is deformed in time by using multiplicative cascades. Although he was able to address fat tails, the non-stationarity in volatility and trending/mean-reversion effects in that model (a summary has been published in Quantitative Finance, refer to his series Mandelbrot (2001, I-IV), he also, in Mandelbrot (2001-III), sketched out a vision of "cartoons", i.e., a fractal trend compositions scheme (by using midpoint replacement techniques) constituting what he called a fractal market. From this viewing angle and particularly supported by his models, he concluded that there are no efficient markets, as assumed by his former student Eugene Fama in Fama (1970). Although many authors after Mandelbrot, and particularly Lo (1998), have shown that there is a long-term dependence of asset returns (violating the weak form of the efficient market hypothesis), there are counter arguments claiming that no one can exploit these dependencies structurally over long time horizons. Particularly, the size and value effect, e.g., reported in Fama (2012), are said to represent a higher insolvency or illiquidity risk. In that regard, the momentum effect, originally reported in Jegadeesh (1993) and also confirmed in Fama (2008) earlier, is of special importance, because it is reported by using simple management rules that leads to structural outperforming portfolios in terms of risk and return compared to a market index.

Recently, in Berghorn (2015), we were able to show that the momentum effect is caused by trends in asset price data. Trends, as discussed, e.g., in Maass (2003), are not mathematically defined in general and the term „trend“ is often used ambiguously. In Berghorn (2015) we used a special wavelet trend decomposition scheme to model price data into up- and downward trends. Particularly, we were able to show that, in accordance with Mandelbrot's scaling analysis, so defined trends in price data are scaled with respect to the granularity level of analysis of that particular wavelet decomposition scheme. Furthermore, the average trend sizes, as computed by the decomposition scheme, are following a power law. When we compared the power law exponents (measuring the average trend sizes) of random processes and the real world data, we were able to show that real world data structurally exhibits higher exponents. By mathematical experiments, we showed that the higher (so-called momentum) exponent (in real world data) is causing the momentum effect. Because the momentum strategy did not exhibit higher risks and had a significant 
higher return, we concluded that there are no efficient markets because the independence of asset returns is nonexistent, and momentum strategies provide simple mechanisms to generate excess returns.

In the following, we will revisit some key components of Berghorn (2015). We use these to establish additional Monte Carlo simulations. Particularly, we test certain random process models by comparing the outcome of a classic momentum strategy with what we have observed with real world data. We will show, in particular, that nonstationary random walks (with piecewise varying drifts and volatilities) do not model momentum well. We will then use trend bootstrapping based on a recursive trend decomposition introduced in Berghorn (2015) and show that this type models the momentum effect quite well. We will analyse the trend statistics of that decomposition and will model price data as being constructed by a superimposition of trends. Under this model (and after taking the logarithm of the prices), the slopes and the sizes of the trends are drawn from a lognormal distribution. This is a natural extension of continuous returns and allows us to derive an analytical form for this type of process. We compare this construction and verify that it (although technically different) fulfils Mandelbrot's vision of fractal markets. This so-called Mandelbrot Market-Model describes the mathematical characteristics enabling the momentum effect. Additionally, we show that market data under this model is wilder than what is usually assumed. Particularly, the shortfall risks of a buy-and-hold investment are significantly higher when we use this model compared to classical approaches. In that regard, we conclude that this model is better suited than classical approaches in literature, such as random walk or fractional brownian motion. Consequently, and in accordance with Mandelbrot's analysis, markets are wilder and exhibit trending (modelled by that model) and, therefore, cannot be efficient.

\section{Trend Momentum Revisited}

In this section, we revisit the main results of Berghorn (2015). In particular, we use a classical long-only momentum strategy for the subsequent experiments. Based on a market's assets, this strategy is executed every month:

1. Calculate the historical 1-year performance of each individual asset in a market.

2. Rank the assets according to their performance from high (performance) to low.

3. Switch/rebalance the portfolio to the $10 \%$ decile of the best performing assets.

4. Subtract a transaction fee of $0,1 \%$ of the portfolio volume.

\subsection{Market Data}

As in Berghorn (2015), we use the Prime-Standard market of the regulated Frankfurter Wertpapierbörse in Germany because this segment has the highest transparency rules. This market is also a prerequisite for companies listed in major market indices such as DAX (major 30 companies in Germany), MDAX (mid-cap segment), TecDAX (technology segment) and SDAX (small cap segment). For our analysis, we use the assets listed in these indexes for mid-April 2014. We use the average price index as a market proxy. Dividend payments are not modelled in the following simulations, although they provide a significant contribution to major indices such as DAX and MDAX. Additionally, we do not correct for delisted companies (survivorship bias) because these have an adverse effect on performance calculations (see Berghorn (2015) for an example). The data is provided by Lenz und Partner AG, a subsidiary of the VWD group.

\subsection{Performance Characteristics}

As listed in Appendix 1, the key performance numbers of this scheme show significant outperformance when compared to the market proxy. The excess return is approximately $19 \%$ p.a., whereas the maximal drawdown is approximately $50 \%$ (maximal drawdown of the market is approximately $65 \%$ ). This risk-adjusted return is $257 \%$ better compared with the market proxy. In particular, the latter characteristic is used to evaluate the Monte Carlo simulations in the remainder of this work.

\subsection{Trend Momentum}

By using a specialised wavelet transform in Berghorn (2015), we were able to model data into up- and downward trends. The granularity of this trend decomposition scheme is defined by the wavelet scale (an example is given in Figure 1 below). 


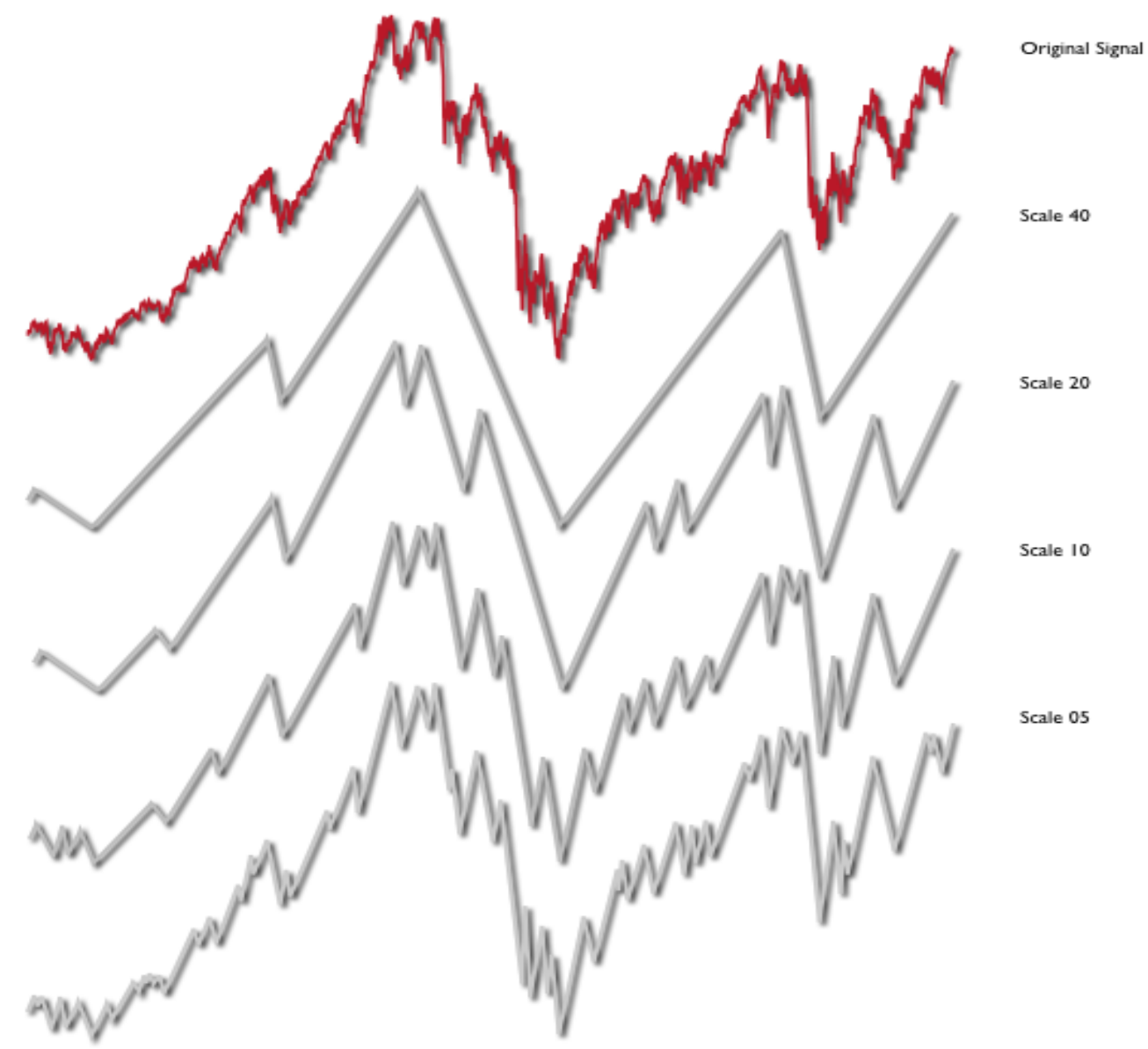

Figure 1. A specialized wavelet transform allows the partitioning of a signal (DAX Performance-Index from 2004 to 2012) into upward and downward trends

The detail of the analysis is parametrized by the wavelet scale. Smaller scales allow the modeling of finer (more detailed) trend structures.

More specifically, let $W_{T}(a, \cdot): S \rightarrow M_{a}$ be the wavelet trend decomposition as depicted in figure 1 above at a positive scale (detail) $a \in \mathbb{R}^{+}$. This decomposition maps a continuous signal $S \in L^{2}(\mathbb{R})$ ( $S$ measurable and $|S|^{2}$ integrable) onto a discrete set of indices. These indices refer to the ordered positions $M_{a}=\left\{m_{0}^{a}, \ldots, m_{n}^{a}\right\}$, $m_{i}^{a} \in \mathbb{R}, m_{0}^{a}<m_{1}^{a} \ldots<m_{n}^{a}$ and $i=\{0, \ldots, n\}$ of local maxima or minima. Between these reversals, we can model a trend segment by a linear function which can be written as

$$
T(S, t, \delta, \Delta, x)=\left\{\begin{array}{cc}
S(t)+\delta \cdot\left(\frac{x-t}{\Delta}\right) & t \leq x<t+\Delta \\
0 & \text { otherwise }
\end{array}\right\}
$$

where $t \in \mathbb{R}$ denotes the time point, $\delta \in \mathbb{R}$ denotes the slope of the trend segment and $\Delta \in \mathbb{R}^{+}$is the size of the trend segment. More specifically, if we have two local extrema at positions $m_{i}^{a}$ and $m_{i+1}^{a}$, we define the $i$-th trend segment as 


$$
T_{i}^{a}\left(S, M_{a}, x\right):=T\left(S, m_{i}^{a}, \frac{S\left(m_{i+1}^{a}\right)-S\left(m_{i}^{a}\right)}{m_{i+1}^{a}-m_{i}^{a}}, m_{i+1}^{a}-m_{i}^{a}, x\right)
$$

Based on the wavelet decomposition, the local extrema are alternating at some scale $a>0$, i.e. a local minimum is followed by a local maximum and vice versa. Having this notation, we can now approximate a signal $S$ by a linear trend model for some positive scale $a \in \mathbb{R}^{+}$:

$$
T_{a}(\cdot)=\sum_{i} T_{i}^{a}(S, M, \cdot)
$$

In the discrete case, where we have a time series

$$
F=\left\{f_{0}, \ldots f_{k}\right\}
$$

of equidistant (daily) sampling points $\{0, \ldots, k\}$ with price values $f_{j}>0, f_{i} \in \mathbb{R}$ we can define the daily (continuous) return by $r_{i}=\ln \left(f_{i}\right)-\ln \left(f_{i-1}\right)$ for $i=\{1, \ldots, k\}$. Consequently, if we have a set of reversals $M_{a}=\left\{m_{0}^{a}, \ldots, m_{n}^{a}\right\}$, we can estimate the (daily) trend drift by

$$
\overline{\mu_{r, a}(l)}=\frac{1}{m_{l+1}^{a}-m_{l}^{a}} \sum_{k=m_{l}^{a}}^{k<m_{l+1}^{a}} r_{k} \text {, i.e. }
$$

averaging the returns on the segment. The trend drift is calculated for all segments $l=\{0, \ldots, n-1\}$. The trend volatility $\sqrt{\overline{\sigma_{r, a}^{2}(l)}}$ is being estimated by

$$
\overline{\sigma_{r, a}^{2}(l)}=\frac{1}{m_{l+1}^{a}-m_{l}^{a}-1} \sum_{k=m_{l}^{a}}^{k<m_{l+1}^{a}}\left(r_{k}-\overline{\mu_{r, a}(l)}\right)^{2}
$$

The average trend size is being calculated by averaging $m_{l+1}^{a}-m_{l}^{a}$ for all $l$.

In Berghorn (2015), we were able to show that there is a dependency between the wavelet scale (granularity level) and the average trend size, which can be verified in the next Figure 2. 


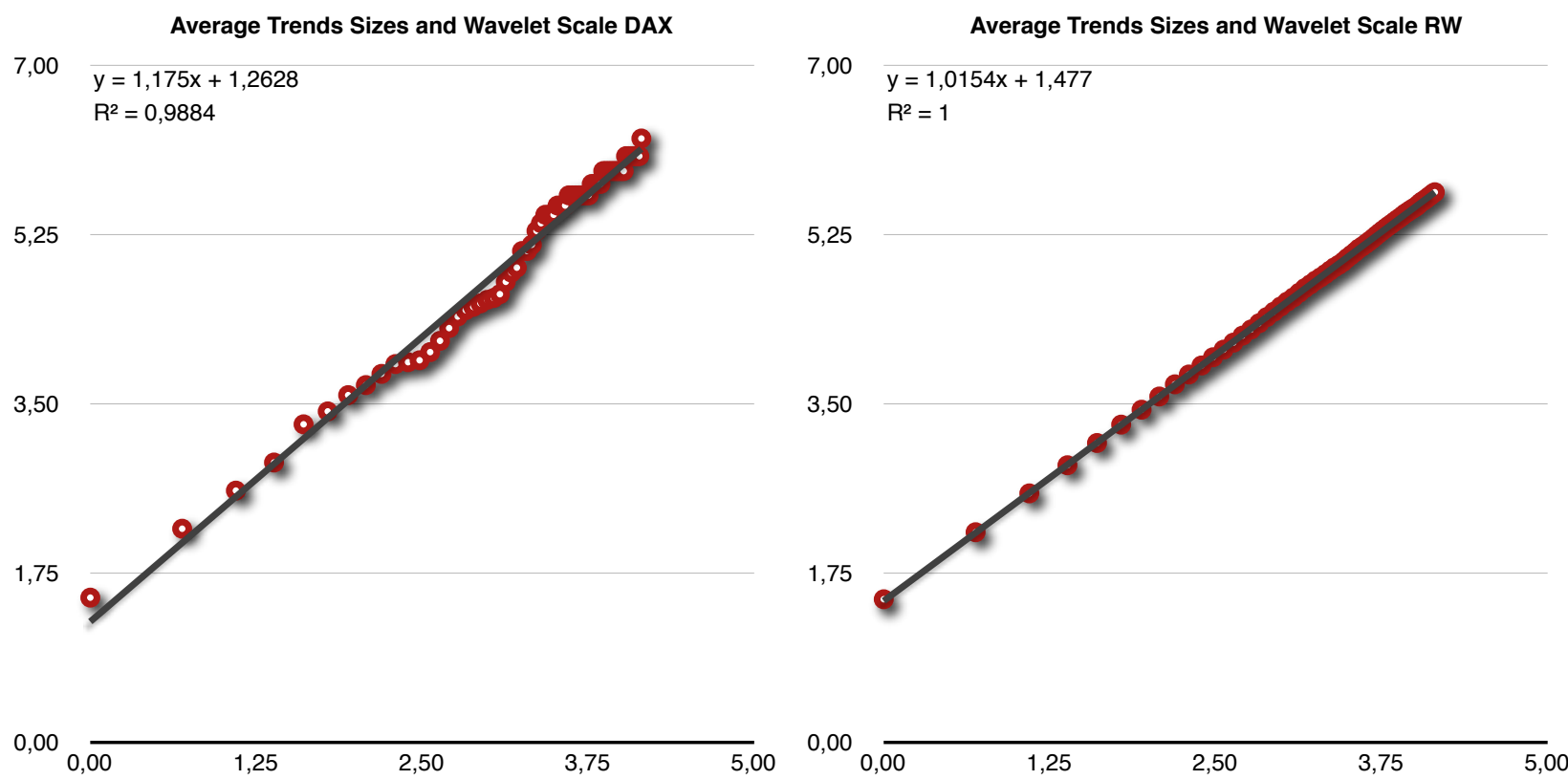

Figure 2

In this figure, we compare, via log-log plot (wavelet scale on the $\mathrm{x}$-axis), the scaling properties of the average trend sizes of the real-world data DAX Performance-Index (1994-2013) to random walks. Specifically, we measure drift and volatility over that period and generate 100 time series using the random walk model with the same size as our original time series (corresponding to 2000 years). As can be observed by this comparison, the exponent of the scaling law in the log-log plot is significantly higher for the real-world data than it is for the random walk counterpart.

Trends under this model do not follow fixed time scales, i.e., for any given wavelet scale, we will observe varying trend sizes (measured in days). Consequently, we report the average trend sizes observed (corresponding to a level of detail described by the wavelet scale). This combination of parameters (average trend size and wavelet scale) follows a scaling law, more specifically, a power-law. Mathematically, let $Y_{\Delta, a}$ be the random variable of trend sizes, then the expected value follows a scaling law, i.e.

$$
E\left(Y_{\Delta, a}\right) \sim a^{\mathfrak{M}} E\left(Y_{\Delta}\right)
$$

where $a \in \mathbb{R}^{+}$and $\mathfrak{M}>0$ denotes the so called "momentum exponent". This signals fractal characteristics. As shown in Berghorn (2015), random processes represented by random walks (geometric Brownian motion) and fractional Brownian motion have so-called momentum exponents close to one ( $\mathfrak{M} \sim 1)$. Real world data exhibits significantly larger momentum exponents $\mathfrak{M}>1$. These larger momentum exponents are the building blocks of momentum portfolios, as can be shown in the following Figure 3: 


\section{Momentum Exponent (Positive Trends) vs. Days Allocated}

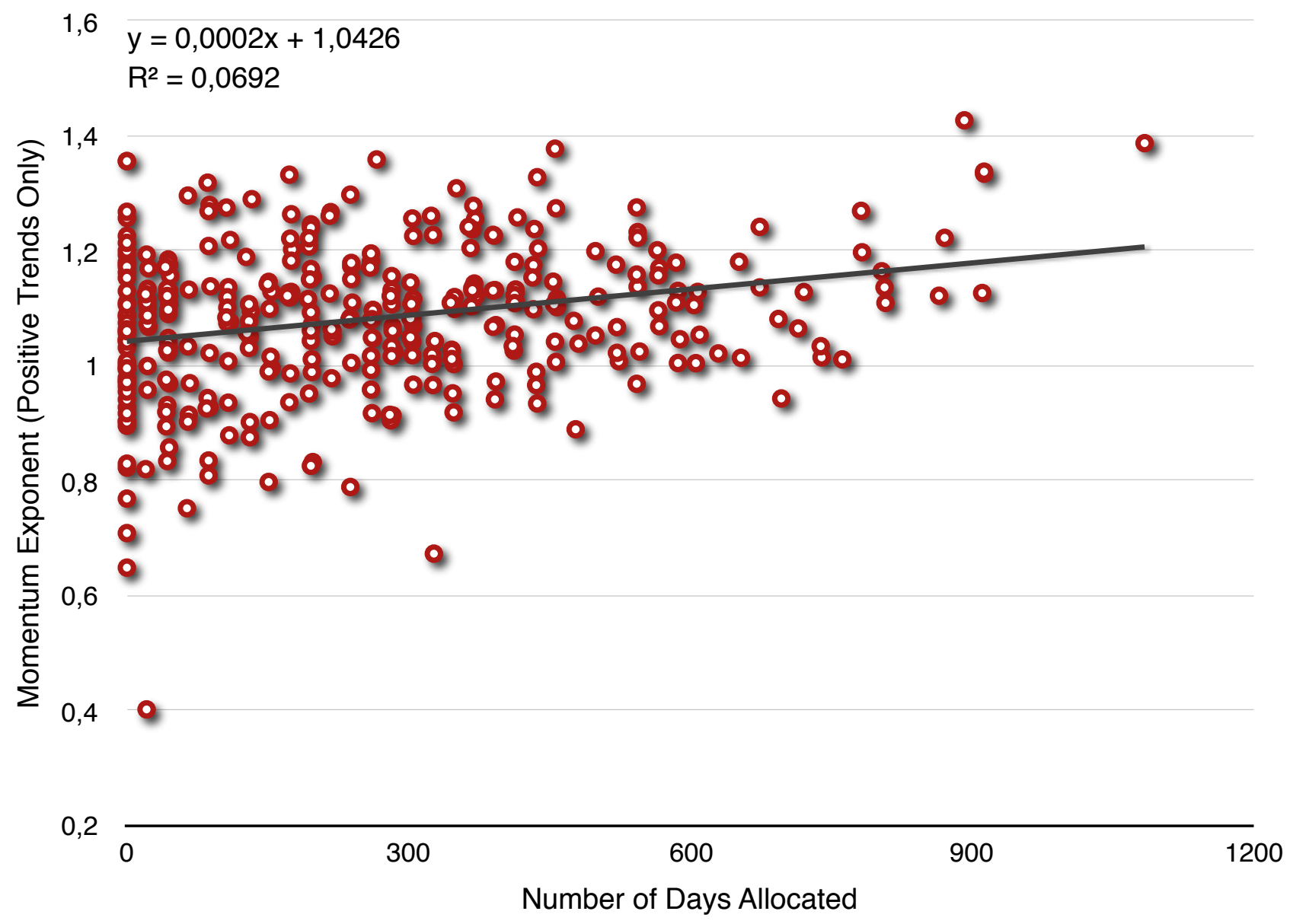

Figure 3

This figure shows the statistical dependency of momentum exponents and the allocation to the momentum portfolio in number of days. We used the classical momentum strategy (long only) on the Prime-Standard market data and calculated for each asset the scaling characteristics of the average trend sizes (momentum exponent) for upward trends. We compare this exponent with the number of days this asset is allocated in the momentum portfolio. As can be observed visually, there is a statistical dependency of the allocation duration and the momentum exponent.

Statistically: The higher the momentum exponent (calculated using upward trends only) for an individual asset is, the longer (on average) the asset will reside in the momentum portfolio. Not all assets with a high momentum exponent are allocated, because an allocation depends on the past performance over one year. The steepness of trends is preferred over the size of trends.

The second characteristic of the wavelet trend decomposition is "visibility". Because the wavelet transform is a convolution (or averaging process), only if these are sharp, steep movements or coarse structures, these structures are visible on a granularity level or a wavelet scale. In terms of trends, these manifest as the "visibility law", i.e., the scaling law describes the product of the average absolute drift and the average trend size in terms of the wavelet scale, as shown in the following Figure 4: 
Plot of $\ln ($ Average Absolute Drift) $x \ln ($ Average Trend Size) vs. In( Wavelet Scale )

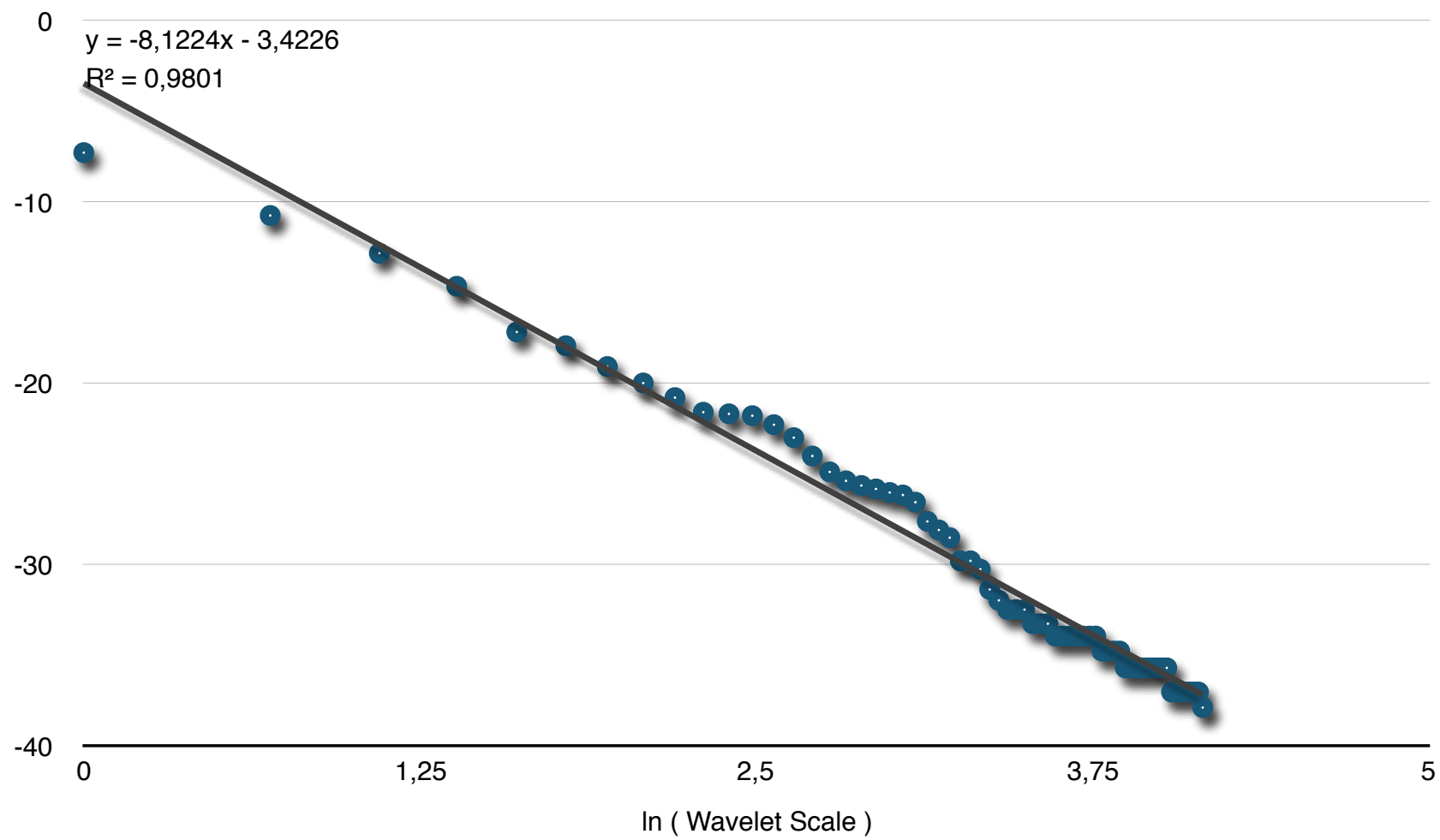

Figure 4

This figure depicts the scaling properties of average absolute trend drift times the average trend drift in a log-log plot for the DAX Performance-Index. Structures observed on a specific wavelet scale are having either (on average) a high drift or are long movements.

Mathematically, let $X_{\delta, a}$ be the random variable representing the trend slope, then there is a relationship between $X_{\delta, a}$ and the trend size represented by $Y_{\Delta, a}$. The ,visibility law“ describes this relationship and we will consider this dependency by defining $\widetilde{X_{\delta, a}}=X_{\delta, a} \mid Y_{\Delta, a}$ in subsequent sections. This dependent variable $\widetilde{X_{\delta, a}}$ is being modeled by using the covariance $\operatorname{COV}\left(X_{\delta, a}, Y_{\Delta, a}\right)$.

\subsection{Monte Carlo Simulations}

In this section, we will repeat a portion of the experiments originally developed in Berghorn (2015). Particularly, we will use three different asset pricing models to generate random price data. This random data will be used to execute the classical momentum strategy. In contrast with Berghorn (2015), we will not use the same time interval for all assets. To amplify deterministic effects, such as new emission of assets, for each individual stock of the Prime Standard, we will use the exact historic time records (on a daily basis in the period 2000 - 2013). These time records will be filled with price data generated by a random process.

We use three proxies for modelling the data used by the Monte Carlo simulation. First, we generate random walks (or geometric Brownian motions) for every asset, in which we estimate the drift (deterministic trend) and the volatility based on the price data in the interval 2000 to 2013. Second, we use classical bootstrapping, in which we store the returns observed for each individual asset during the period 2000 to 2013, and in which we draw historic returns by using a uniform distribution to select a particular return from this historic collection. Finally, we estimate the Hurst exponent (using the $\mathrm{R} / \mathrm{S}$ analysis) over the same period and generate fractional Brownian motions using the drift, volatility and the Hurst exponent. 
For each individual asset (using the historic time record in the period 2000 to 2013), we generate one outcome based on the three methods. On that collection of outcomes, we execute the classical (long only) momentum strategy. We count any collection of price data in which we have a momentum strategy yielding a higher risk-adjusted return than observed in the market ( $257 \%$ better than the market proxy generated based on that random data).

As can be verified in Table 1, independent processes, such as random walks or bootstrapping, do not model the momentum effect well:

Table 1

Monte Carlo Simulations 2004-2013 - I

Monte Carlo Simulation

\begin{tabular}{lr}
\hline Bootstrapping & $11 \%$ \\
\hline Random Walk & $10 \%$ \\
\hline Fractional Brownian Motion & $48 \%$
\end{tabular}

For the historic window 2000-2013, the daily drift, the daily volatility, and the Hurst exponent are measured for each individual asset (341 assets). These characteristics are used to create random outcomes using Monte Carlo simulations of the period 2000-2013, in which we use the original dates of the historic prices recorded. We also collect the log returns for every asset from which we draw uniformly (bootstrapping). We apply the classical momentum strategy on these outcomes for the shorter period 2004-2013 (10 years). The whole generation process is repeated 100 times, yielding a momentum simulation of 1,000 years in total. We measure the amount of strategy iterations in which we observe in a momentum strategy a higher outperformance (i.e., a better risk-adjusted performance than the $257 \%$ observed in the real data). We re-run the experiment three times using different generation methods. As observed from this table, bootstrapping has $11 \%$ of iterations with higher outperformance compared with the real data. For random walks, we observe $10 \%$ of the iterations with higher outperformance, and fractional Brownian motions generate $48 \%$ iterations, with better outperformance observed in the true market.

\subsection{Conclusion}

As shown in table A above, serial independence of price data (as assumed by the weak form of the efficient market theory), as expected, does not explain the momentum effect. Because there is also a vast amount of literature available (documenting the momentum effect for several markets and time horizons), and momentum strategies, as we have observed, do not exhibit higher risks, one should carefully reconsider whether the independence claim (and the efficient market hypothesis) is truly valid.

The R/S analysis used here (and documented in Berghorn (2015)) reveals that over 90\% of the assets used in the above example exhibit trending (described by a Hurst exponent $>0.5$ ). Consequently, the chance that we observe the momentum effect as strong as we have observed in the historic data is approximately $50 \%$.

However, fractional Brownian motions do not show high momentum exponents (as documented in Berghorn (2015)). We can only conclude that this model is a better model (with respect to the momentum effect) than the serial independent models.

Consequently, we are in need of the development of new models that reconcile these effects (describe the momentum effect and have a momentum exponent similar to what has been observed in the market).

In the next section, we will show that a classic non-stationary geometric Brownian motion, common in literature, does not model the momentum effect well either. Based on that result, we will revisit an alternative price construction based on a recursive decomposition shown in Berghorn (2015).

\section{Trend Models}

The stochastic processes described above share one common characteristic. At the core of the process an individual price return is drawn (either by bootstrapping or drawing from a normal distribution). By design, the fractional 
Brownian motion process multiplies a decaying (anti-auto-correlation or) auto-correlation structure onto this independent process. As described in Hull (2014), it is common to assume non-stationarity in these types of processes, i.e., to assume varying drift and volatility. Particularly, the latter, originally introduced by Engle (1982), is assumed to model heteroscedasticity for the ARCH type of models. In Berghorn (2015), we used a certain wavelet scale to replicate a momentum strategy that evaluates the performance over one year; therefore, it is reasonable to assume that momentum strategies exploit trends at coarser scales than what we used in that experiment.

\subsection{Classical Non-Stationary Trend-Model}

To verify this claim, we use the following experiment. Given the momentum strategy (using a 10\% decile, i.e., 34 assets) of the Prime Standard segment, we will determine the granularity level or wavelet scale that best represents the trends exploited by the momentum strategy.

This means that we decompose, for a given wavelet scale, every asset of the Prime Standard into its linear upward and downward trend segments. The asset pricing series is then replaced by that trend model that represents trends on that given scale. For these bare trend replications of all assets, we execute the aforementioned momentum strategy and compare the excess return to the strategy on real world data. We then optimise the wavelet scale, i.e., we select that wavelet scale for which the excess return of the momentum strategy using the trend representation of the underlying assets is closest to the results using real world data. To avoid ambiguities, we select the lowest wavelets scale for that procedure.

The following Figure 5 represents the outcome of this experiment.

\section{Prime Standard Comparison of Market Proxy to Momentum Strategy on Trend-Models}

$900 \%$

- Averaged Prices (Market Proxy) Cumulative Return

- Classic Momentum Strategy Cumulative Return using Trend-Model at Scale 17

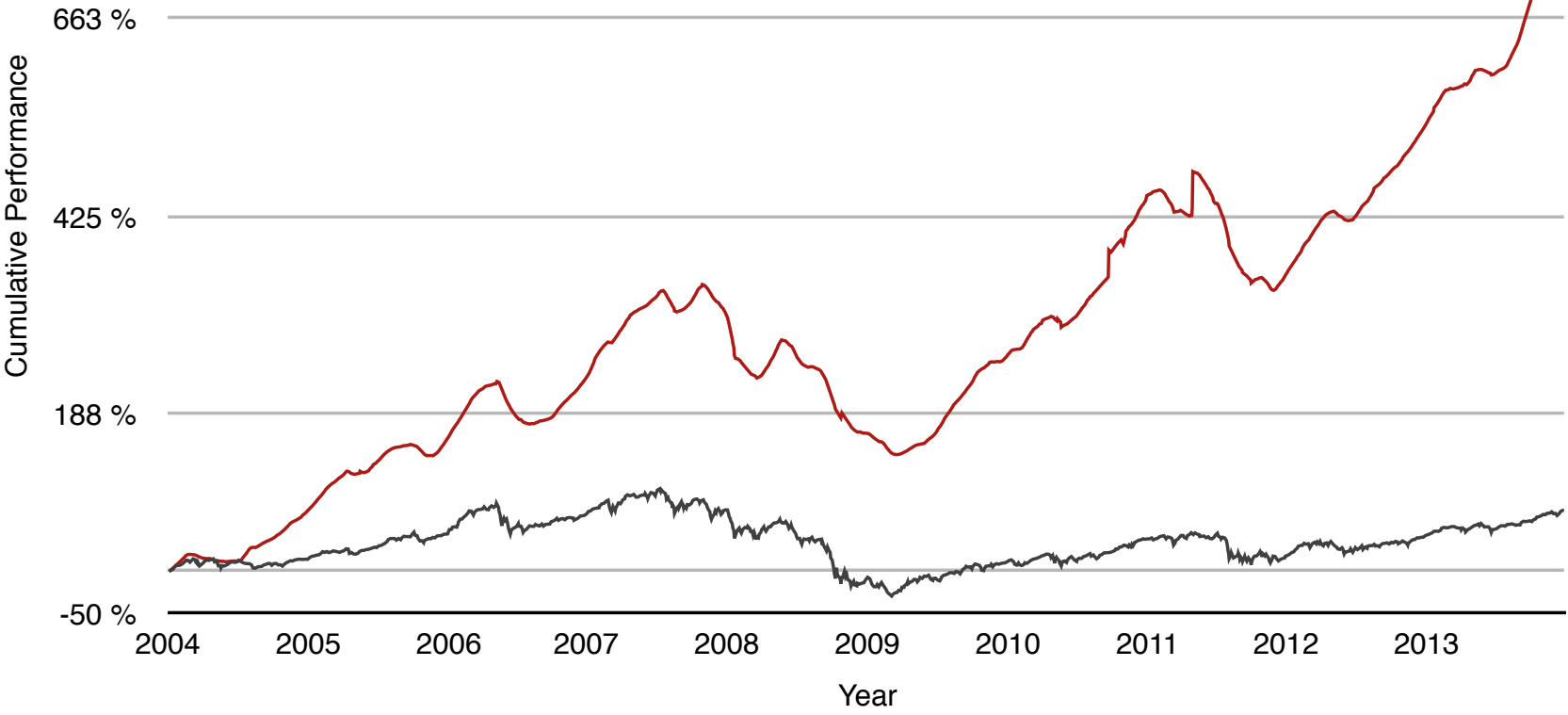

Figure 5

This figure depicts the results of a momentum strategy using a linear upward and downward trend models for the underlying assets of the market. The scale for the trend models are chosen so that the excess return is close to the original observation using real data. Using the determined wavelet scale 17 yield following characteristics: The excess return (compared to the market proxy using price averaging) is 19,31\% p.a.. The maximal drawdown is $-45,99 \%$. The daily volatility is $0,33 \%$ compared with $1,27 \%$ of the original classical momentum strategy (using real data). 
As shown by this figure, the linear trend model (substituting the original asset data) at a wavelet scale of 17 replicates the excess return observed in the strategy using real data (see Appendix 1). Although we use piecewise linear trends (without any random fluctuation) for the asset data, the resulting daily volatility of the momentum strategy is $0,33 \%$ compared with $1,27 \%$ for the original experiment. This means that approximately $25 \%$ of the volatility observed in momentum strategies originates from underlying (in this example, deterministic) trends.

Can we now model data as an underlying signal plus noise as is common in engineering? We will answer this by establishing a non-stationary random walk and using that model for Monte Carlo simulations of the classical momentum strategy:

Let us consider that we have a wavelet trend decomposition at scale 17 (as used above) for every asset of the underlying market Prime Standard. For every trend segment, we can estimate the drift and the volatility characterising the original signal in that trend segment. Thus, we can model non-stationary random walks. From the original random walk model (with drift), we replace the deterministic trend (or drift) by the trend-wise determined drifts using the wavelet trend decomposition. We model these trends based on historical observations. Additionally, for every trend segment, we use the estimated volatility in that segment. This construction leads to piecewise defined random walks. Each piece is represented by a trend segment characterised by the drift and volatility of that segment.

Using this model, we generate (as done previously) 100 realisations of the entire market for the time period 2000 to 2013 and execute the classical momentum strategy on every market realisation. We then count all realisations for which the momentum strategy yields a higher risk-adjusted return compared with what we measured originally in real data. As shown in the next Table 2, this model also does not model momentum well:

Table 2

Monte Carlo Simulations 2004-2013 - II

\begin{tabular}{lr} 
Monte Carlo Simulation & Higher Outperformance \\
\hline Bootstrapping & $11 \%$ \\
\hline Random Walk & $10 \%$ \\
\hline Fractional Brownian Motion & $48 \%$ \\
\hline Fixed Scale Trend Model & $9 \%$
\end{tabular}

For the historic window 2000-2013 we decompose each individual asset (341 assets) into trends using the wavelet scale 17. For each trend segment, we estimate the drift and volatility. We now generate trend wise random walks, where we use the drift and volatility associated to the trend segment seen on wavelet scale 17 . We apply the classical momentum strategy on these outcomes for the shorter period 2004-2013 (10 years). The whole generation process is repeated 100 times, yielding a momentum simulation of 1,000 years in total. We measure the amount of strategy iterations where we see in a momentum strategy a higher outperformance (i.e., a better risk-adjusted performance than the $257 \%$ observed in the real data). Only $9 \%$ of these realizations have a higher risk-adjusted return compared to the original data.

Although this model does not explain momentum, one must analyse the statistics of the trends determined by the wavelet trend decomposition. Because momentum strategies detect these linear trend segments, the distribution of the trend size is of special importance. As observed later and also in Appendix VI.2, the log normal distribution well describes the trend sizes across various scales. This means that we can assume trends to be drawn from a lognormal process. Consequently, trends at a certain visibility level (wavelet scale) can have varying sizes; in particular, the lognormal distribution is fat-tailed. However, trends at a certain wavelet scale contain trends at coarser scales. To reconcile for these effects, we will use a dyadic, recursive trend decomposition that is similar to the fast dyadic wavelet transform widely used in signal processing. 


\subsection{Bootstrapping with Trends}

In this section, we will use the dyadic trend decomposition, originally introduced in Berghorn (2015). Although technically different, this will implement the vision of Mandelbrot regarding fractal markets. The motivation to apply this special form of decomposition originates from two sides. First, trends detected at a scale by construction will always contain trends that belong to coarser scales. To obtain trends that belong characteristically to a single scale, we should remove coarser trends at higher scales. Second, this scheme is similar to dyadic wavelet transforms, which also operate recursively. In Figure 6, we revisit the decomposition introduced in Berghorn (2015):

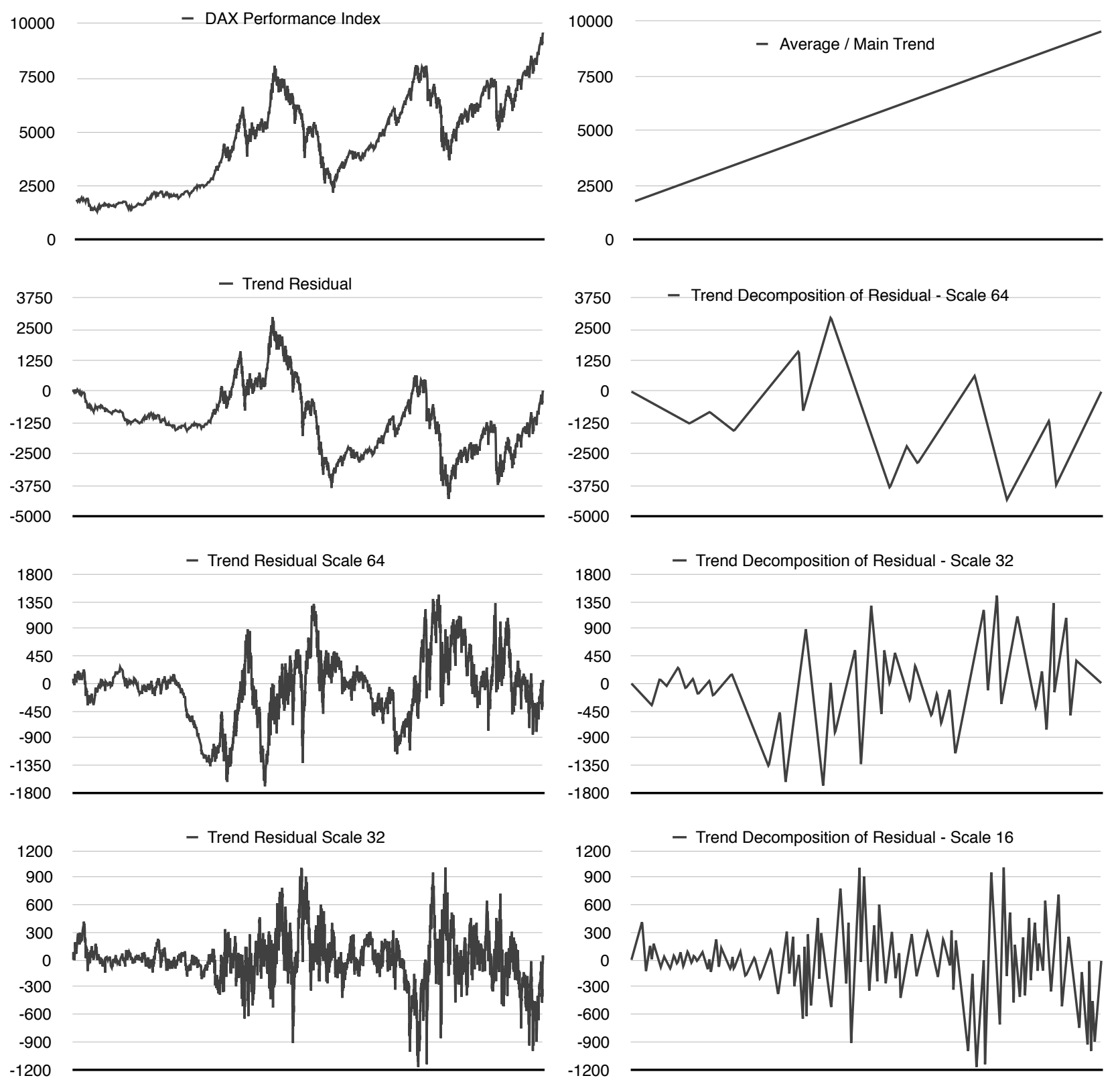



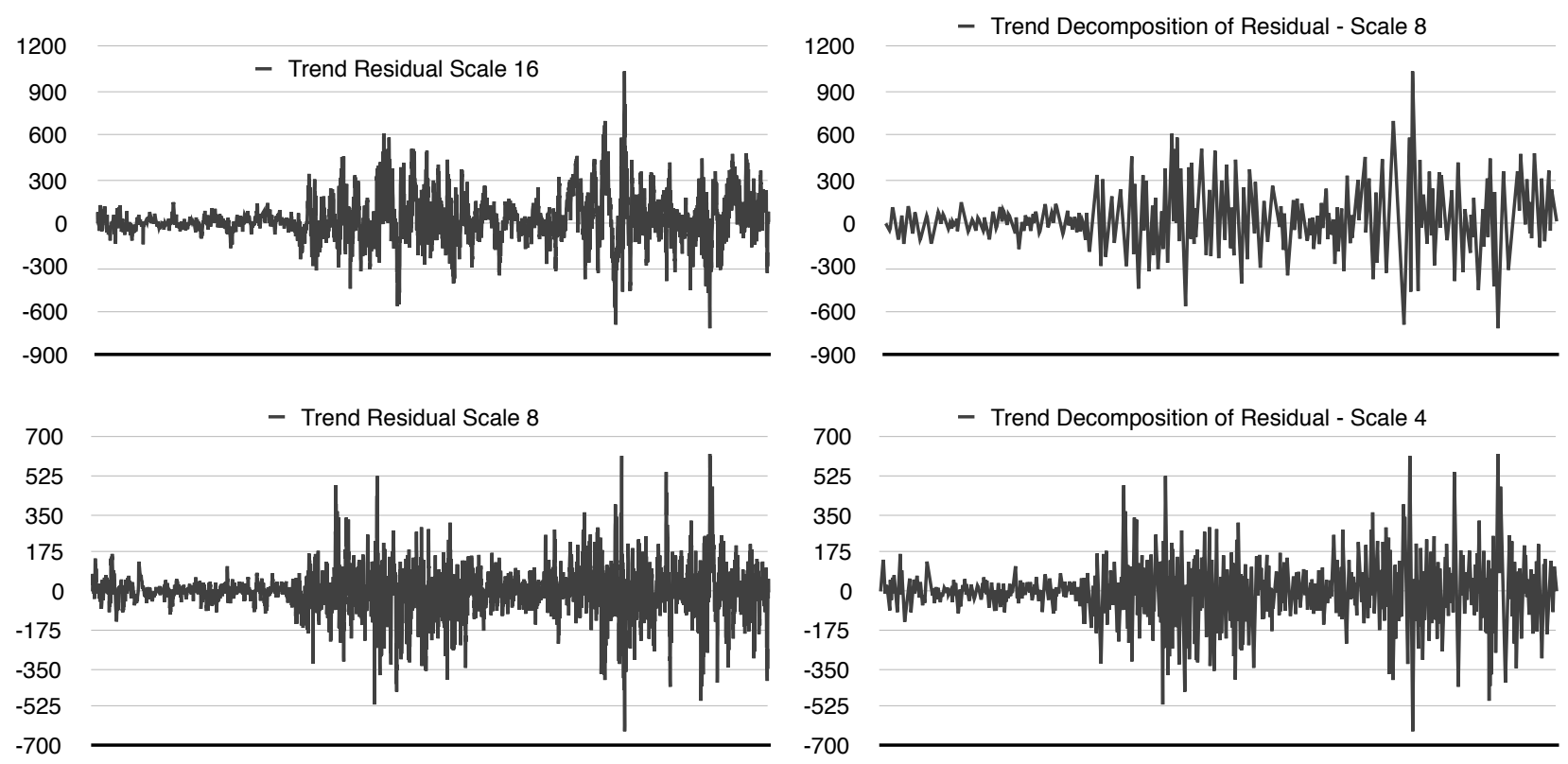

Figure 6

This figure depicts the multi-scale characteristics of the wavelet trend decomposition scheme for the extended period 1990 to 2013 (24 years) for the DAX Performance-Index. The top left graph shows the original time series. Next to it (top right), we show the main trend. The next rows use a successive de-trending. From the original data series (top left), we remove the major trend (top right) and list the residual (de-trended time series) on the left in the next column. Consequently, the average of this residual is zero. To the right (second row), we apply a trend decomposition on scale 64 on the former residual and depict the major trends observed at that scale. In the third row (left), we depict the residual of the previous de-trending process. The whole approach is continued recursively to scale 4 . As can be observed from the graphs, we have, since 2000, a very different trending characteristic with increased fluctuations on low scales. This is in accordance with heteroscedasticity/volatility clusters observed in finance data. Additionally, these phenomena are spread across several scales, showing the self-similarity of these phenomena. Finally, at scale 4, the recursive decomposition process is nearly "converging," i.e., no new information is gained by decomposing further.

Conceptually, we are replacing a random walk by a recursive, multi scale, and trend-based random process. In the initial step, we use the price series and calculate the log prices.

Let $f \in L^{2}(\mathbb{R})$ be a price function. Given a compact support $C$ (as realistic in the discrete case) we define

$$
f_{c}(x)=\left\{\begin{array}{cc}
f(x)>0 & x \in C \\
0 & x \notin C
\end{array}\right\} .
$$

We transform $f$ by applying the logarithm, i.e. we define

$$
g_{C}(x):=\left\{\begin{array}{cc}
\ln (f(x)) & x \in C \\
0 & x \notin C
\end{array}\right\} .
$$

This allows us to consider trends (of varying sizes) as trend returns similar to continuous returns (which correspond to trends with a time scale of one day for end-of-day data). Analogous to a classic geometric Brownian motion, the main trend, starting from the initial log price and ending with the last log price, is the deterministic trend.

We define the main trend by the compact support $C=\left[c_{1}, c_{2}\right]$ with $c_{1}, c_{2} \in \mathbb{R}$ and $c_{1}<c_{2}$ and 


$$
\tau_{C}(x)=\left\{\begin{array}{cc}
\frac{f_{c}\left(c_{2}\right)-f_{c}\left(c_{1}\right)}{c_{2}-c_{1}} \cdot x & x \in C \\
0 & x \notin C
\end{array}\right\} .
$$

After having removed the main trend, i.e. having the difference $d_{C}^{\infty}:=g_{C}-\tau_{C}$, we obtain the residual fluctuating around zero. On that residual, we apply the wavelet trend decomposition at a high scale, e.g., 64, and store all trends. Trends in that scheme are characterised by two parameters (at a wavelet scale $a$ ): The slope of the trend (in the following denoted by $X_{\delta, a}$ ) and the size in days (in the following denoted by $Y_{\Delta, a}$ ). Because we have used the logarithm of the entire time series $f$ in the beginning, these two characteristics correspond to the return over the size of the trend (slope times size). The continuous return (or log return) commonly used is a special case of this approach because it corresponds to a slope with a fixed size of one day.

We now apply the wavelet trend decomposition scheme on $d_{C}^{\infty}$ and obtain the reversals

$$
W_{T}\left(a^{*}, d_{C}^{\infty}\right)=M_{a^{*}}=\left\{m_{0}^{a^{*}}, \ldots, m_{n}^{a^{*}}\right\} \text { for an initial scale } a^{*} .
$$

After decomposing the first residual $d_{C}^{\infty}$ (after removing the main trend) into trends, we again remove this trend structure from the residual and reduce the wavelet scale for the decomposition of that residual by a factor of two. We then calculate the trends on that finer residual using the finer scale and proceed with this entire process until we reach scale 4 .

Mathematically, we model with these reversals the function $d_{C}^{\infty}$ as upward and downward trends

$$
T_{a^{*}}^{d^{\infty}}(\cdot)=\sum_{i} T_{i}^{a^{*}}\left(d_{C}^{\infty}, M_{a^{*}} \cdot\right)
$$

We remove from $d_{C}^{\infty}$ the trend structure $T_{a^{*}}^{d^{\infty}}(\cdot)$ and define the next „,de-structured“ difference

$$
d_{C}^{a^{*}}:=d_{C}^{\infty}-T_{a^{*}}^{d^{\infty}}
$$

Finally we repeat this process for dyadic wavelet scales, i.e. let $A=\left\{a^{*}, \frac{a^{*}}{2}, \frac{a^{*}}{4}, \ldots, \frac{a^{*}}{2^{v}}\right\}$ be an arbitrary sequence of dyadic and successively finer wavelet scales and denote $a_{i}$ the $i$-th element of that sequence, then we can define the recursive procedure

$$
d_{C}^{a_{i}}:=d_{C}^{a_{i-1}}-T_{a_{i}}^{d^{a_{i-1}}}
$$

I.e. we remove from the de-structured signal successively finer trend details.

As shown in Figure 6, on a scale of 4, we reach a type of "convergence", i.e., subdividing even further does not generate new structures.

Because we used a trend subtraction scheme across scales and stored all trends (by their two characteristics slope and size) in the order of decomposition, we can easily reconstruct the original price series algorithmically as follows:

Start at scale 4 and at time 0 . Draw the first trend from the previously stored list and calculate a linear trend segment ranging from the origin to the point described by the size and slope times size. Draw the next trend from the stored list and concat the next linear trend segment described by the next size and the next slope to the previous one. Continue this concatenation process until we reach the end of our original time series.

We repeat this construction process for all scales $8,16,32$. We then have linear trend models for all these scales. Because we established the process earlier by the subtraction of trends from the residual, we can revert the process by simply adding the trend representations on the aforementioned scales. If we also add the main trend removed in the beginning as well as the residuum (after removing all trends) and use the exponential function on the result, we can reconstruct the original price series. 
Because the trend characteristics (slope and size in the log space) are a natural extension of continuous returns (slope with fixed size of one), it is also straightforward to apply classical bootstrapping techniques usually used for continuous returns for the trend process described above. To do that, we need to introduce randomness during the reconstruction process described above.

More specifically, if we draw trends stored per scale during the analysis process by a uniform distributed selection process, and not in the order of occurrence, we obtain trend bootstrapping.

The next Figure 7 shows realisations of this trend bootstrapping process based on the DAX Performance-Index from 1990 to 2013.

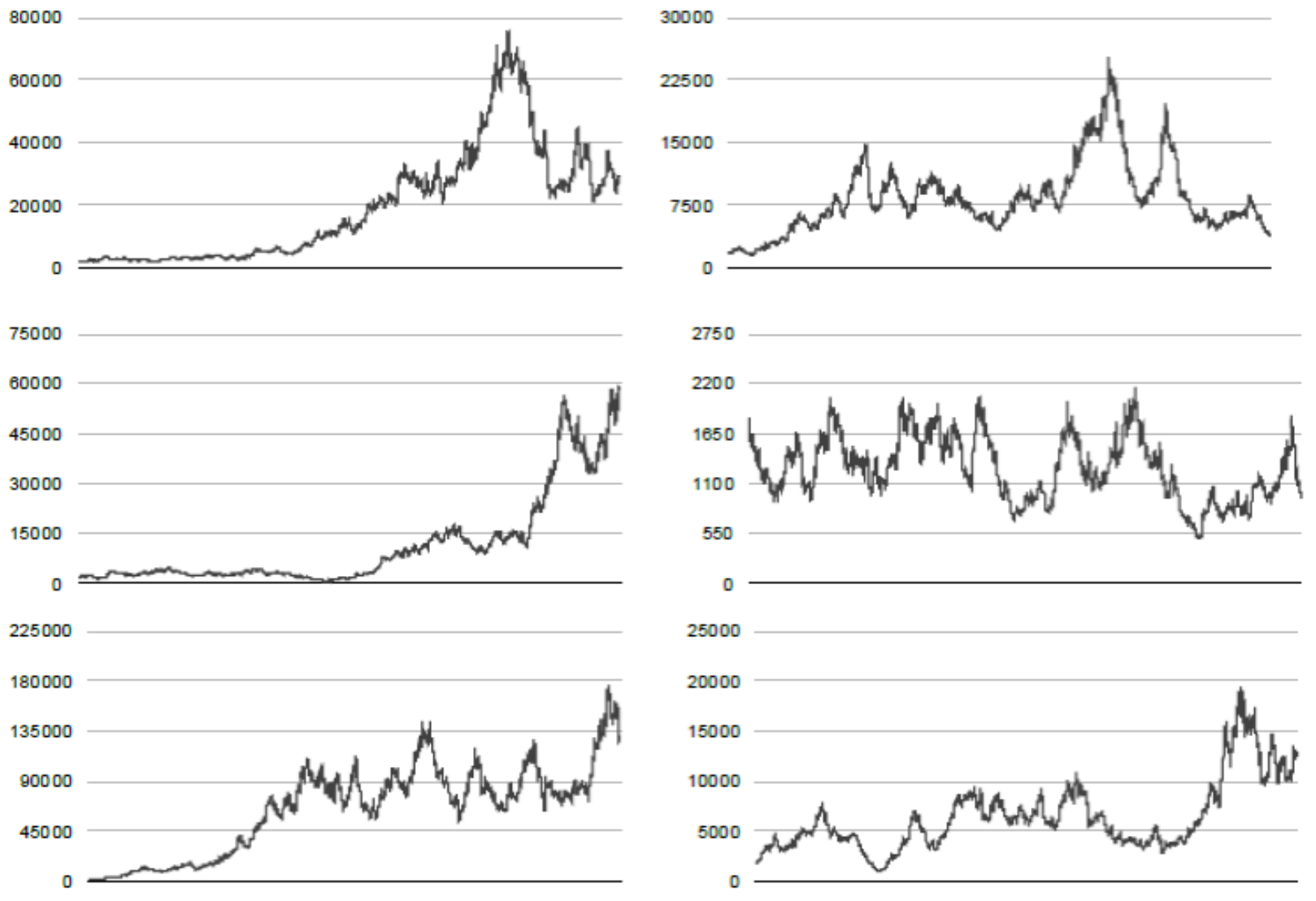

Figure 7

We show different realizations based on the trend bootstrapping process representing the DAX Performance-Index 1990 to 2013 (24 years). During the recursive analysis process (after taking the log of the prices) we store all trends observed in a list. Every trend is described by a tuple of slope and size. Using a uniform distribution, we select per scale an arbitrary trend from that list. This linear trend segment is concatenated to the previous drawn trends (or the origin in the beginning). The different scales are added after this bootstrapping process. Finally, we add the main trend (removed during the analysis phase from the beginning), add via bootstrapping the residuum, and apply the exponential function to the result.

If we were only using the main trend and the first residuum in this procedure and would model this residuum as normal distributed with some variance, we would create a random walk with (deterministic trend) drift.

Is this process then random or deterministic? From the outside, it appears as a random process; from the inside, it appears as a superimposition of deterministic trends in random order. As we will verify in the next table, this bootstrapping technique models momentum quite well. 
Table 3

Monte Carlo Simulations 2004-2013 - III

Monte Carlo Simulation

\begin{tabular}{lr}
\hline Bootstrapping & $11 \%$ \\
\hline Random Walk & $10 \%$ \\
\hline Fractional Brownian Motion & $48 \%$ \\
\hline Fixed Scale Trend Model & $75 \%$ \\
\hline Trend Bootstrapping & \\
& For the historic window 2000-2013 we previously used, we calculate for every individual asset of the Prime \\
Standard the recursive, dyadic trend decomposition on the log prices as described above. For every scale \\
representing a residual, we bootstrap trend characteristics (slope and size) and add the results. Finally, we add the \\
main trend, add the final residuum (after removing all trends) via bootstrapping, and apply the exponential function \\
for all prices. We again generate 100 realizations representing 1000 years of momentum strategy (as previously \\
done). In 75\% of the cases we will see a risk-adjusted return higher or equal to what we have observed in the original \\
data. Consequently, this model is representing the momentum effect.
\end{tabular}

If we model every asset of the Prime Standard using this technique, we will have roughly a $75 \%$ chance to observe the momentum effect (or stronger) that we have originally observed the real data (Note 1).

\subsection{Comments}

According to this model, there are no efficient markets because prices are not independent. This is in accordance with literature documenting the momentum effect in many markets and also supported by outcomes from Lo's specification test for the US (Lo (1998)). Mandelbrot has criticised this assumption of independence from early on and proposed, with his fractional Brownian motions, a different model. As we have documented in Berghorn (2015), the German Prime Standard market has Hurst exponents higher than 0.5 for nearly every asset. Although technically different, this trend bootstrapping approach described above also implements Mandelbrot's vision of fractal markets summarised in Mandelbrot (2001-III). His cartoons illustrating the fractal (recursive) nature of markets are based on mid-point replacement techniques, which were used to generate fractal landscapes, refer to Peitgen (1988). As documented there, mid-point replacement techniques are not well suited for approximating fractional Brownian motion because they generate visual artefacts when used to render landscape sceneries. In the context of fractal price series, these cartoons (understood also as an approximation for fractional Brownian motion) are also not well suited for another reason: If a main trend is being drawn or set (e.g., by a random process), the midpoint replacement scheme will not alter the beginning and the end of the trend. Mandelbrot intended to have his models used in Monte Carlo techniques, e.g., to estimate risks. The midpoint replacement approach would be ill suited for this purpose because, e.g., a shortfall risk estimate, would depend solely on the generation of this main trend.

However, the generation process described above fulfils his vision; therefore, we call this the Mandelbrot MarketModel. Instead of the midpoint replacement technique, we introduced an additive process in which trends are superimposed in a recursive manner by sub trends on lower scales. Technically, we also reverted the reconstruction process to circumvent the drawback of midpoint replacement with respect to the start and the end of the time series. If we then were able to replace the bootstrapped trend characteristics by a model and would allow rescaling, we would be able to generate a fractal market. This is shown in the next section.

\subsection{Analytical form of the Mandelbrot Market-Model}

As we will show in this section, the trend size $\left(Y_{\Delta, a}\right)$ estimated in the log space are best modelled using lognormal distributions. We estimate the mean and standard deviation of the trend size in days for the scales 4,8 16 and 32 . For this purpose, we optimise the goodness-of-fit of the log-normal distribution parameters using maximum-likelihood estimates for every individual wavelet scale (Cullen 1999). We test these results using the null hypothesis; the null 
hypothesis tested is that the trend size $\left(Y_{\Delta, a}\right)$ originates from the log-normal distribution with the estimated mean and standard deviation using the Kolmogorov-Smirnov test. The test statistics and p-values are provided in Table 4:

Table 4

Null Hypothesis : Trend-Size is Log-Normal Distributed

\begin{tabular}{lccccc} 
Decomposition Method & $\mathbf{4}$ & $\mathbf{8}$ & $\mathbf{1 6}$ & $\mathbf{3 2}$ & \\
\hline Single Scale & 0.0799 & 0.0702 & 0.1333 & 0.1154 & Statistic \\
\hline & $(0.231)$ & $(0.8328)$ & $(0.5176)$ & $(0.9966)$ & $\mathrm{p}$-Value \\
\hline Single Scale RW & 0.0384 & 0.0294 & 0.0288 & 0.0242 & Statistic \\
\hline & $(0.0000)$ & $(0.0000)$ & $(0.001954)$ & $(0.1955)$ & $\mathrm{p}$-Value \\
\hline Recursive Decomposition & 0.0489 & 0.0645 & 0.0722 & 0.1875 & Statistic \\
\hline & $(0.6553)$ & $(0.7572)$ & $(0.9623)$ & $(0.6272)$ & $\mathrm{p}$-Value \\
\hline Recursive Decomposition RW & 0.059 & 0.0435 & 0.0299 & 0.0243 & Statistic \\
\hline & $(0.0000)$ & $(0.0000)$ & $(0.0002)$ & $(0.1722)$ & $\mathrm{p}$-Value
\end{tabular}

Using the DAX Performance-Index (1990-2013) as well as the corresponding random walk model (using 100 realization with estimated drift and volatility over this period), we decompose the resulting price series using both trend decomposition methods described above. With "Single Scale" we denote the decomposition method per fixed scale. "Recursive Decomposition" denotes the recursive decomposition scheme at scales 4,8,16,32 as described above. The addendum "RW" denotes that a corresponding random walk model was used to collect the trend size statistic. The columns 4,8,16,32 denote the outcome of the Kolmogorov-Smirnov test, where we use the null hypothesis of trend sizes drawn from a lognormal distribution. We list per test the test statistics as well as the pvalue. As can be observed from these results, we fail to reject the null hypothesis of lognormal distributions at a $5 \%$ level of significance.

We fail to reject the null hypothesis of lognormal distribution at the $5 \%$ level of significance for both the single scale and recursive decomposition models. Conversely, the test for the random walk models (single scale and recursive decomposition) reject the null hypothesis, except for scale 32. Furthermore, we were unable to define another fitting distribution for the random walk models.

We conclude that higher scales (corresponding to coarser trend structures) tend to have a better fit with the lognormal distribution than lower scales (corresponding to finer structures). These effects may be explained by a longterm "return to the mean" effect.

Hence, we conclude that trend size $\left(Y_{\Delta, a}\right)$ generated by the Mandelbrot Market-Model are not random and cannot be explained by a hidden random walk effect with trends and drift.

However, and as described above, trend sizes $\left(Y_{\Delta, a}\right)$ and trend slopes $\left(X_{\delta, 1}\right)$ are not independent processes because of the "visibility law" depicted in figure 4 . Because structures are visible at a given scale, if they are either sharp movements or coarse longer lasting structures, we must model the conditional probability between the two characteristics. More specifically, we introduced $\widetilde{X_{\delta, 1}}=X_{\delta, 1} \mid Y_{\Delta, 1}$ reflecting the "visibility law" earlier.

In the following experiment, we use the general trend decomposition and reconstruction scheme used for the trend bootstrapping approach described above. Instead of drawing the tuple trend size and slope from existing statistics, we model the trend size $\left(Y_{\Delta, a}\right)$ and the slope $\left(X_{\delta, 1}\right)$ as lognormal distributed. From a formal standpoint we create the Mandelbrot Market-Model by an initial white noise process 


$$
M_{B}^{0} \sim N\left(\mu_{0}, \sigma_{0}^{2}\right)
$$

for some given $\mu_{0}$ and $\sigma_{0}^{2}$. We generate the next level by

$$
M_{B}^{1}=M_{B}^{0}+T_{B}^{1}
$$

where $T_{B}^{1}$ corresponds to a trend structure at scale $a=1$, which we will define below. This trend structure is generated by realizing from the lognormal distributed random variable $X_{\delta, 1}$ (trend slope) and the $Y_{\Delta, 1}$ (trend size), and considering the dependence $\widetilde{X_{\delta, 1}}=X_{\delta, 1} \mid Y_{\Delta, 1}$. More broadly: Let

$$
\widetilde{x_{1, \delta, i}, \ldots, \widetilde{x_{n, \delta, i}}}=x_{1, \delta, i}\left|y_{1, \Delta, i}, \ldots, x_{n, \delta, i}\right| y_{n, \Delta, i}
$$

be realizations of $\widetilde{X_{\delta, i}}$ given the realizations $y_{1, \Delta, i}, \ldots, y_{n, \Delta, i}$ of $Y_{\Delta, i}$, i.e. the realizations of trend slopes and trend sizes of the $i$-th level of the construction process. Then we can define the trend structure $T_{B}^{i}$ as

$$
T_{B}^{i}(t)=\left\{\begin{array}{c}
\vdots \\
\sum_{k<l}\left((-1)^{k} \cdot \widetilde{x_{k, \delta, i}} \cdot y_{k, \Delta, i}\right)+(-1)^{l} \cdot \widetilde{x_{l, \delta, i}} \cdot\left(t-\sum_{k<l} y_{k, \Delta, i}\right) \\
\text { for } \quad \sum_{k<l} y_{k, \Delta, i} \leq t<\sum_{k \leq l} y_{k, \Delta, i} \\
\vdots
\end{array}\right\}
$$

In other words, $T_{B}^{i}$ is the concatenation of trend realizations. In algorithmic terms: we have to determine for a given time point $t$ the index $l$ so that $t$ falls into the $l$-th trend realization interval. We then determine the prior trend paths by cumulatively adding the trend sizes $y_{k, \Delta, i}$ times the realized slopes $\widetilde{x_{k, \delta, i}}$ for $k<l$ and concatenate the current trend realization.

Eventually, we have

$$
M_{B}^{2^{i}}=M_{B}^{2^{i-1}}+T_{B}^{i}
$$

for $i \in \mathbb{N}$ which is fractal by definition, since it is recursively defined, uses self-affine trend-distributions and the trend structures $T_{B}^{i}$ have a fractional dimension.

From a practical standpoint, we estimate the covariance matrix between the two characteristics trend size and slope (per wavelet scale) to model their dependency. We distinguish between upward trends and downward trends, i.e., we model both situations separately. Using the statistics of upward trends and downward trends, we calculate the two different covariance matrices for modelling this dependency of size and slope.

As described above, the modelling procedure is initialised by drawing a vector (size and slope) from a normal distribution with zero mean and a variance of one. We then multiply the Choelsky decomposition of the covariance matrix with this realisation and apply the exponential function to the result. Finally, we model the "residual" (after removing trends at successive higher scales) as normal distributed, because of the law of large numbers. The error should be normal distributed, if we had a sufficient large number of samples. This realisation of a trend is concatenated as previously in the bootstrapping scheme. We depict realisations in Appendix IV.2.

As depicted in the next table, this analytical form of the Mandelbrot Market-Model is nearly equivalent for modelling momentum as the trend bootstrapping method. 
Table 5

Monte Carlo Simulations 2004-2013 - IV

Monte Carlo Simulation

Higher Outperformance

\begin{tabular}{lr}
\hline Bootstrapping & $11 \%$ \\
\hline Random Walk & $10 \%$ \\
\hline Fractional Brownian Motion & $48 \%$ \\
\hline Fixed Scale Trend Model & $9 \%$ \\
\hline Trend Bootstrapping & $75 \%$ \\
\hline Analytical Fractal Trend Model & $66 \%$
\end{tabular}

As for Table 3 we are using the recursive trend decomposition to model the asset data used for the Monte Carlo simulations of the momentum effect. Instead of bootstrapping the trends observed in the original data, we use lognormal models to generate the trend slopes and sizes. Note, that due to the "visibility law" both characteristics are dependent. The conditional probability is modeled by using a covariance estimate between both characteristics. This model, which has an analytical form, is nearly equivalent in describing the momentum effect compared to the trend bootstrapping method.

\subsection{Comments}

Although this (analytical) model has slightly less descriptive capabilities than the trend bootstrapping version, it shows that the concepts of fractal trend decompositions as envisioned by Mandelbrot actually worked. Similar to the trend decompositions at various scales as depicted in Figure 2, we are able to obtain scaling laws using the recursive approach sketched above. The next figure depicts these scaling laws for the trend sizes and the slopes (using a loglog-plot) across the dyadic scales 4,8,16, and 32.

Average Trends Sizes and Wavelet Scale DAX

6

$y=1,2104 x+0,8797$

$\mathrm{R}^{2}=0,9928$

5

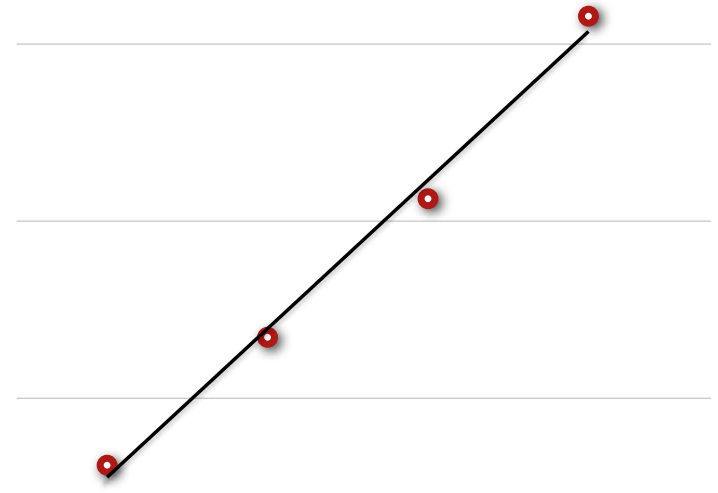

2

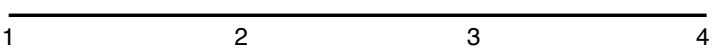

Average Trends Sizes and Wavelet Scale RW

6

$y=1,0998 x+1,0873$

$R^{2}=0,9981$

5

3

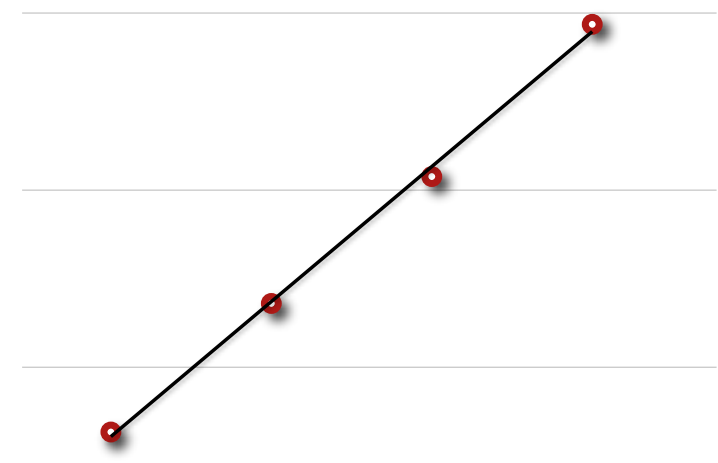

2

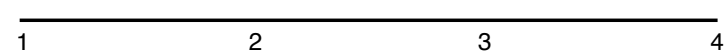



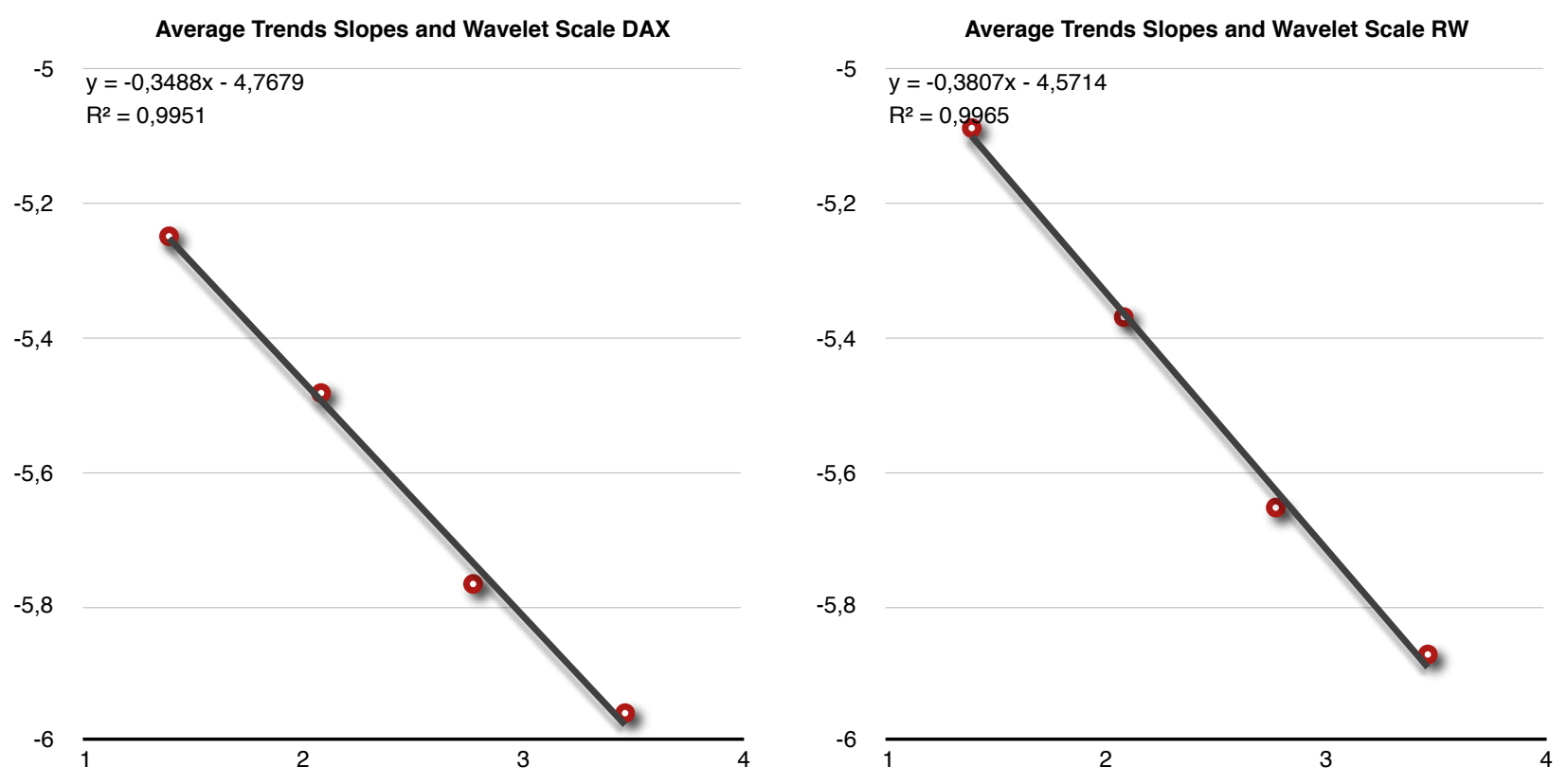

Figure 8

Previously, as in Figure 2, we compare via log-log plot (wavelet scale on the x-axis) the scaling properties of the average trend sizes (top row) and the average trend slopes of the real-world data DAX Performance-Index (19942013) to random walks. Specifically, we measure drift and volatility over that period and generate 100 time series using the random walk model with the same size as our original time series (corresponding to 2000 years). Similar to the single scale decomposition, the momentum exponent representing the scaling behavior of the trend size in the recursive decomposition scheme is significantly higher compared to the random walk model. Both characteristics exhibit scaling laws.

Using these scaling laws above, it is easy to observe that we can generate a fractal market. By starting the construction at an initial wavelet scale, we can draw trends from the conditional distributions described for the trend size and the trend slope. We can then successively construct coarser scales and use the additive process to generate coarser structures. Assuming that the scaling law holds for all higher scales, we can create an infinite process, i.e., we can zoom out arbitrarily and will observe coarser structures that are statistically self affine to the finer detail. Although this process reverses usual fractal constructions schemes (which usually zoom in), it creates a fractal.

Before we conclude, we will analyse Mandelbrot's other major insight that markets are much wilder then generally assumed. Mandelbrot tied this mainly to the fact of fat tailed return distributions. However, as we will show in the next sections, this observation also holds true for trends.

\section{Risk Analysis}

Although a thorough analysis of the risk side of the Mandelbrot Market-Model proposed above is beyond the scope of this work, we want to indicate that these models not only model the momentum effect well, but also indicate a significantly higher risk of assets in general.

To observe this, in the following, we will compare the average trend sizes of upward and downward trends. 
Comparison of Average Trend Sizes DAX Performance-Index (1994 - 2013)

800
- Average Trend Size of Upward Trends
- Average Trend Size of Downward Trends

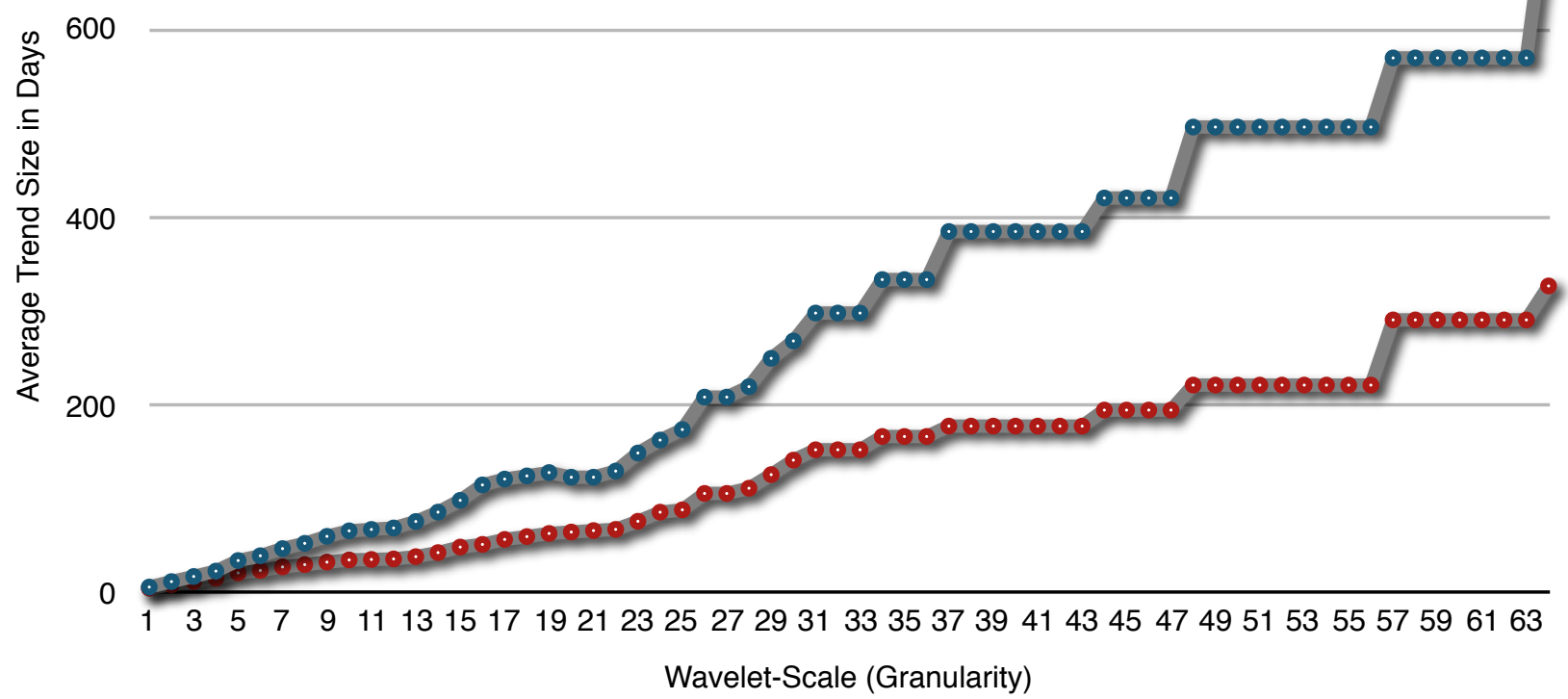

Figure 9

In this figure we compare the average size of upward trends with the average size of downward trends. The underlying index is the DAX Performance-Index from 1994 to 2013 (20 Years). For every granularity level (wavelet scale), we use the trend decomposition and calculate the average trend size observed. As one can easily see, upward trends are on average longer than downward trends, which is (assuming a random walk model with drift) due to the positive drift.

As depicted in Figure 9 above, the average trend size for upward trends is higher across all scales compared with downward trends. If we combine this outcome with the average returns observed in trends, we can clearly identify the higher risk characteristics in the market. 
Comparison of Average Daily Returns DAX Performance-Index (1994 - 2013)

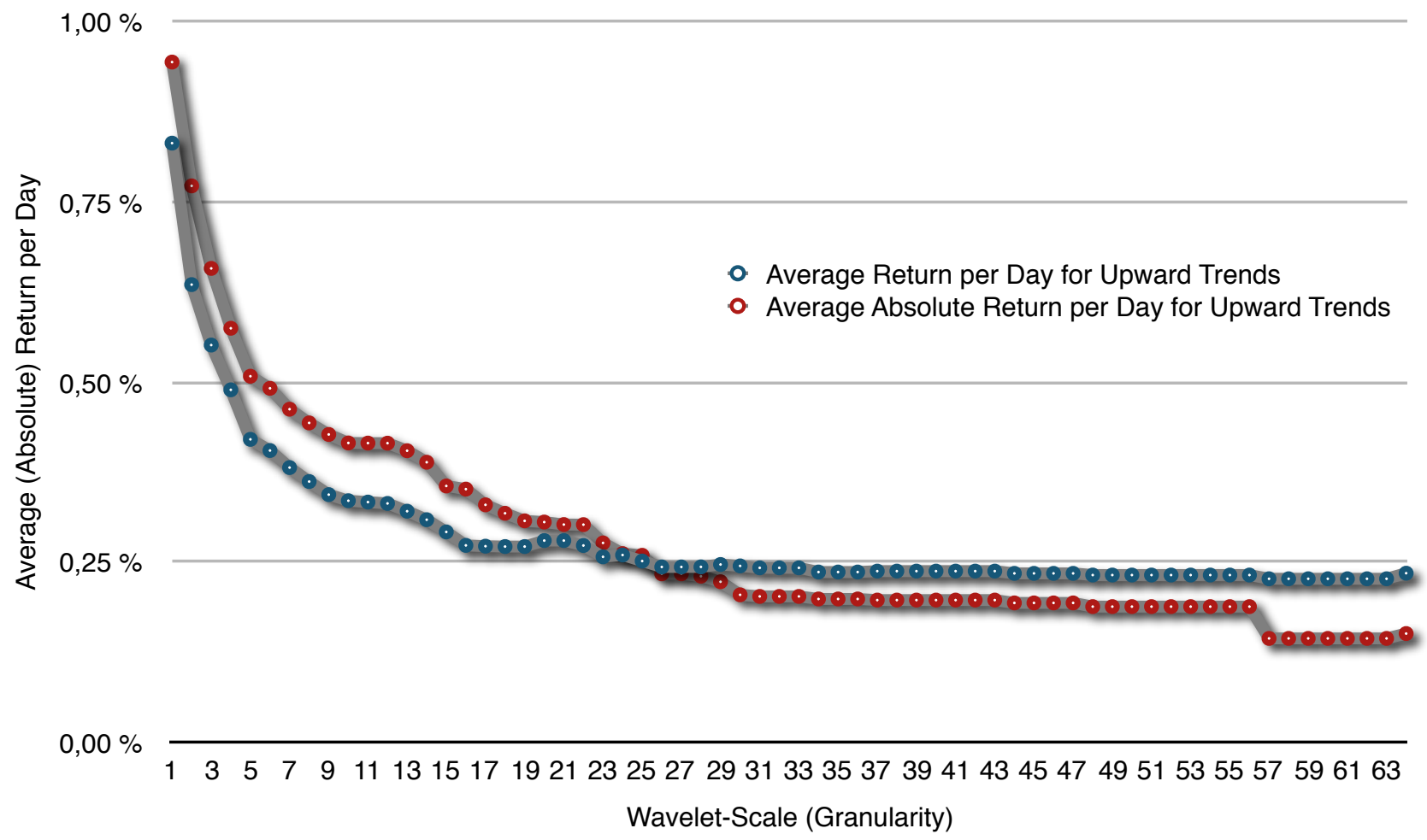

Figure 10

As in Figure 8 we compare the upward trends to the downward trends for the DAX Performance-Index during the time period 1994 to 2013 (20 years). In this figure we depict the average (daily) return for upward trends, i.e. we measure the return for a trend and divide it by the number of days (linear increment). After taking the absolute return value, we do this also for the downward trends. At a wavelet scale of 25 and lower downward trends exhibit higher average returns compared to upward trends.

As depicted in Figure 10, downward trends to a maximum scale of 25 exhibit higher (absolute) returns compared to upward trends. Consequently, downtrends (up to a granularity of 25) are fast and steep movements, where upward trends are in general longer and slower (in terms of steepness). As shown in Berghorn (2015), this also holds true if one compares trends in real world data to random walks. These characteristics carry over to a general shortfall profile. For the final experiment, we use a Monte Carlo simulation, in which we estimate the difference in shortfall risks over a buy-and-hold period of ten years of a random walk model compared with the trend bootstrapping approach. As shown in the Fgure 11, markets are roughly twice as wild under this new model compared with a classic random walk. 


\section{Shortfall Risks Random Walk vs. Trend Bootstrapping (DAX P-I 1994-2013)}

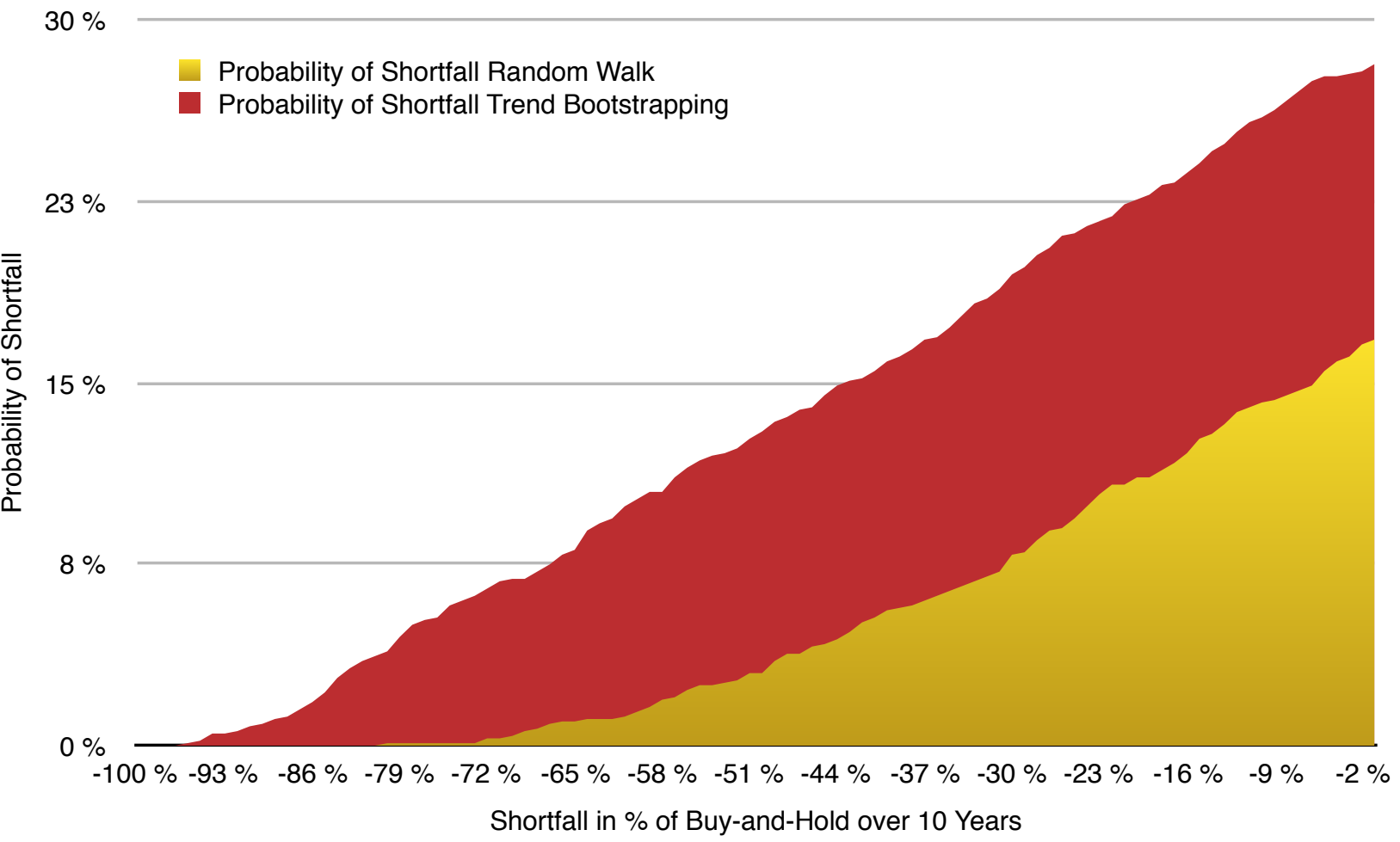

Figure 11

In this figure, we compare the shortfall risk using two different models: The classical random walk model (with drift) and the trend bootstrapping approach introduced in section II. We use the DAX Performance-Index during the period 1994 - 2013 to collect the statistical parameters drift and volatility used for the random walk generation. Although, we could provide an analytical form for the random walk, we will use a Monte Carlo generation to visualize numerical errors. For the trend bootstrapping approach, we analyze the same period. We then draw with both models 100 realizations from these two different processes and measure the conditional probability of seeing a specific loss over a buy-and-hold period of 10 years. The trend bootstrapping approach, in this example, roughly indicates a shortfall risk that is twice as high compared with the random walk model.

Although a more elaborate analysis is beyond the scope of this work, we currently can observe that trends are responsible not only for the reward side (as exploited by momentum strategies), but also contribute heavily to the risk side. Both characteristics have been highlighted by Mandelbrot from early on and have been analysed through Mandelbrot's vision of fractal markets, as summarised in the conclusions below.

\section{Conclusions}

If one wants to summarise Mandelbrot's contributions to the area of finance, one may find two major themes: Markets have long-term memory (as modelled by fractional Brownian motions), and markets are wilder than expected (usually derived by a fat-tail analysis of the returns). In this work, we were able to build on earlier findings presented in Berghorn (2015). These showed that asset data consists of trends, and that the granularity level used for the analysis (wavelet scale) is linked to the average trend sizes via a power law. Based on the scaling analysis of trends and the associated momentum exponent, we were able to show that classical random processes (such as geometric but also fractional Brownian motion) are sub-optimally suited to capture the momentum effect. In this work, we were able to show that the common basis of these processes (drawing a single return to determine the price of the next day) is not well suited. Motivated by our previous results indicating fractal characteristics, we were able to show that Mandelbrot's vision of fractal markets (called cartoons) are a meaningful representation of market data.

Particularly, this vision allows us to model the momentum effect better, but also provides insight into the risk nature of markets. By providing an analytical representation, we were able to provide a model nearly equivalent to the 
bootstrapping technique with respect to the momentum effect. This model assumes trends to be drawn from random processes and superimposed to the final price series. As such, it allows us not to forecast prices with sufficient precision, but enables the implementation of momentum strategies in the long -term.

Although we have not reconciled for (multi scale) heteroscedasticity effects (clearly present), as this is beyond this work, we were previously able to show that markets are wilder not only because of fat tailed return distributions, but also because of trends of various detail scales. In essence, we were able to show two ground beliefs of Benoit Mandelbrot: Markets are fractal and there are no efficient markets.

We conclude with a citation of Mandelbrot (2006):

"His [Newton's] was a very small seed of thought, from which a great forest of science and engineering has grown. My [Mandelbrot's] hope is that, someday, the small seed of multifractal analysis can grow into a fruitful new way of managing the world's money and economy."

\section{Acknowledgements}

We want, particularly, to thank Heinz-Otto Peitgen for his supporting efforts and the many guiding remarks. As a good friend of Benoit Mandelbrot, he shared many of the personal stories of Mandelbrot's life. As do the authors, he believes that Mandelbrot's work deserves much more recognition in finance than in the past.

We also want to thank Christian Jasperneite from M. M. Warburg \& CO (Hamburg, Germany) for reviewing the simulation results. We want to thank Marcel van Leeuwen (DPWT Deutsche Wertpapiertreuhand GmbH, Herzogenaurach, Germany) for his view of the practical side and for the discussion around Mandelbrot's vision. Finally, we want to thank Peter Singer (Fachhochschule Ingolstadt, Germany), who reviewed this work and the simulation results.

\section{References}

Bachelier, L. (1900). Théorie de la Spéculation. Annales scientifiques de l'École Normale Supérieure, 3(17), 21-86.

Berghorn, W. (2015). Trend Momentum. Quantitative Finance, 15(2), 261-284. http://dx.doi.org/ $10.1080 / 14697688.2014 .941912$

Cullen, A. C., \& Frey, H. C. (1999). Probabilistic techniques in exposure assessment. Plenum Press, USA, pp. 81-155.

Dempster, M. A. H., \& Benoit, B. M. (1924-2010). A father of Quantitative Finance. Quantitative Finance, 11(2), 155-156.

Engle, R. F. (1982). Autoregressive Conditional Heteroscedasticity with Estimates of the Variance of United Kingdom Inflation. Econometrica, 50(4), 987-1008.

Fama, E. F. (1970). Efficient capital markets: A review of theory and empirical work. J. Finance, 25(2), 383-417.

Fama, E. F., \& French, K. R. (2008). Dissecting anomalies. J. Finance, 63, 1653-1678.

Fama, E. F., \& French, K. R. (2012). Size, value, and momentum in international stock returns. J. Financial Economics, 105, 457-472.

Hull, J. C. (2014). Options, Futures, and Other Derivatives. Pearson Education.

Jegadeesh, N., \& Titman, S. (1993). Returns to buying winners and sell- ing losers: Implications for stock market efficiency. J. Finance, 48, 65-91.

Lo, A. W., \& MacKinlay, A. C. (1998). Stock market prices do not follow random walks: Evidence from a simple specification test. Rev. Financ. Stud., 1(1), 41-66.

Maass, P., Koehler T., Kalden, J., Costa, R., Parlitz, U., Merkwirth, Ch., \& Wichard, J. (2003). Mathematical methods for forecasting bank transaction data. DFG-Schwerpunktprogramm 1114, Mathematical methods for time series analysis and digital image processing, Preprint 24.

Mandelbrot, B. (1963). The Variation of Certain Speculative Prices. The Journal of Business, 36(4), 394-419. The University of Chicago Press.

Mandelbrot, B. B. (2001). Scaling in financial prices: I. Tails and dependence. Quantitative Finance, 1, 113-123.

Mandelbrot, B. B. (2001). Scaling in financial prices: II. Multifractals and the star equation. Quantitative Finance, 1 , 124-130. 
Mandelbrot, B. B. (2001). Scaling in financial prices: III. Cartoon Brownian motions in multifractal time. Quantitative Finance, 1, 427-440.

Mandelbrot, B. B. (2001). Scaling in financial prices: IV. Multifractal concentration. Quantitative Finance, 1, 641-649.

Mandelbrot, B. B., \& Hudson, R. L. (2006). The (Mis)Behaviour of Markets: A Fractal View of Financial Turbulence. Basic Books, annotated edition edition.

Mandelbrot, B. B., \& Van Ness, J. W. (1968). Fractional Brownian Motions, Fractional Noises and Applications. SIAM Review, 10(4), 422-437. Society for Industrial and Applied Mathematics.

Mandelbrot, B. B., Fisher, A. J., \& Calvet, L. E. (1997). A Multifractal Model of Asset Returns. Cowles Foundation Discussion Paper No. 1164; Sauder School of Business Working Paper. Retrieved from http://ssrn.com/ abstract $=78588$

Peitgen, H-O. (Editor) (1988). The Science of Fractal Images. Springer.

Peitgen, H-O., \& Mandelbrot, B. B. (1924-2010). Science 12, 2010, 330 (6006), 926. https://doi.org/10.1126/ science. 1199471

\section{Note}

Note 1. Using 100 realizations (due to the complexity of the fractional brownian motion) is allowing us to support the central claim of this work. E.g. if we extend the Monte Carlo simulation using 1,000 realizations for random walk and the trend bootstrapping approach, we see similar outcomes. In particular, we see $7.8 \%$ of random walk and $72.1 \%$ of trend bootstrapping realizations above the risk adjusted outperformance seen in the market data. 


\section{Appendix A}

\section{A.1 Classical Momentum-Strategy Lon-Only Prime-Standard 2004 - 2013}

In Berghorn (2015), we showed the following Figure 12 illustrating the momentum effect on the Prime-Standard segment in Germany through the time period 2004-2013.

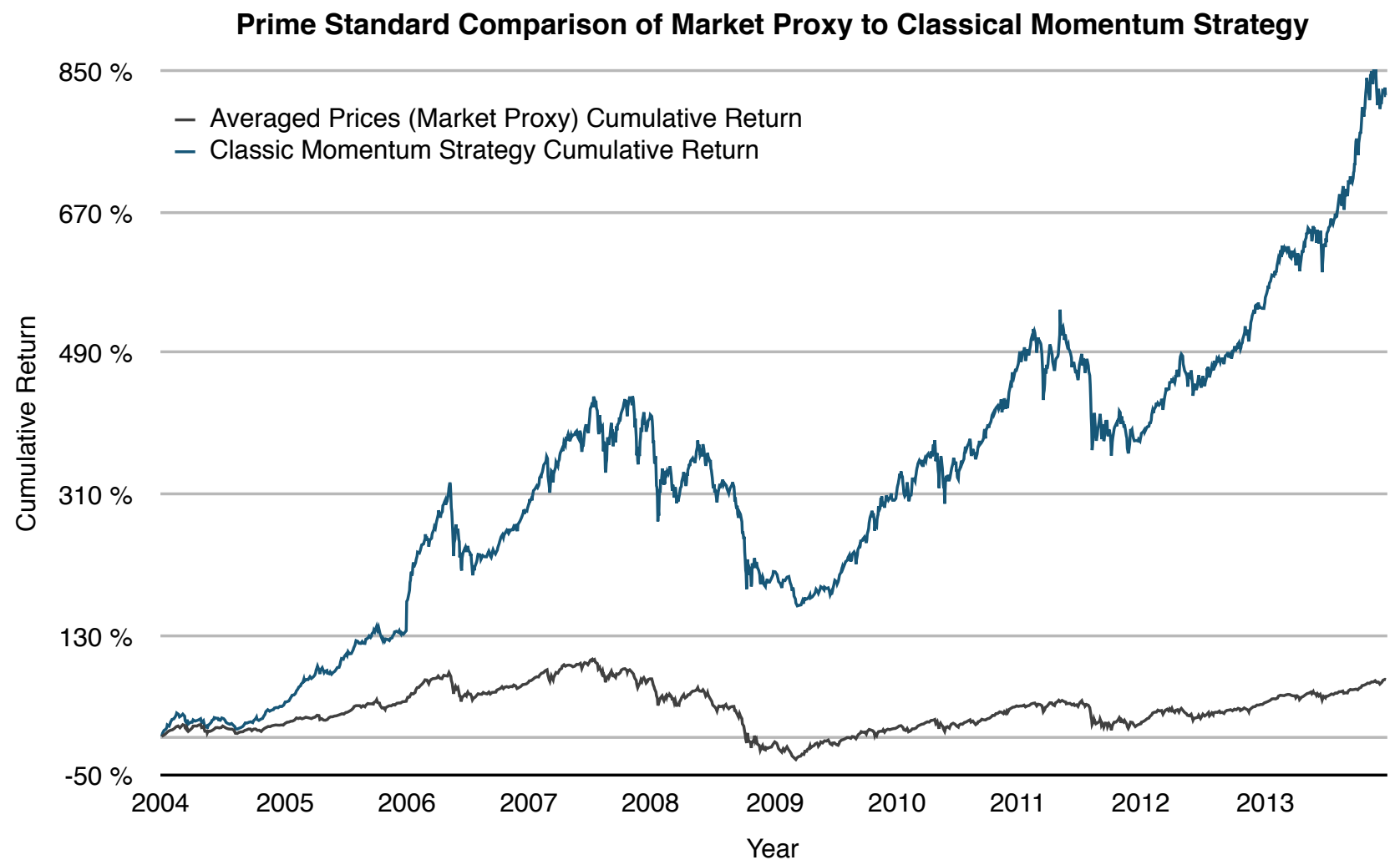

Figure 12

This figure shows cumulative performance charts of the underlying market proxy of the Prime Standard segment (by calculating the averaged prices) compared with the classical momentum strategy using 34 assets (10\% decile) and a 12-month evaluation interval over the period 2004 to 2013 (10 years).

The main characteristics of this strategy were: Excess return of $19,22 \%$ p.a. including monthly transaction costs of $0,1 \%$ per full volume. The maximal drawdown was $-50,33 \%$ compared with a maximal drawdown of $-64,76 \%$ of the market proxy.

\section{A.2 Realisations of the Analytical Mandelbrot Market Model}

Figure 13 depicts realisations of the analytical form of the Mandelbrot Market Model. The construction process is similar to the trend bootstrapping approach described above. In the log space, a trend comprising the characteristics "size" and "slope" are drawn from a lognormal distribution preserving the correlation structure representing the "visibility law". The residual is being modelled as normal distributed (in the log space). This is repeated for all recursive scales, and the result is summed and transformed to the original price space. 

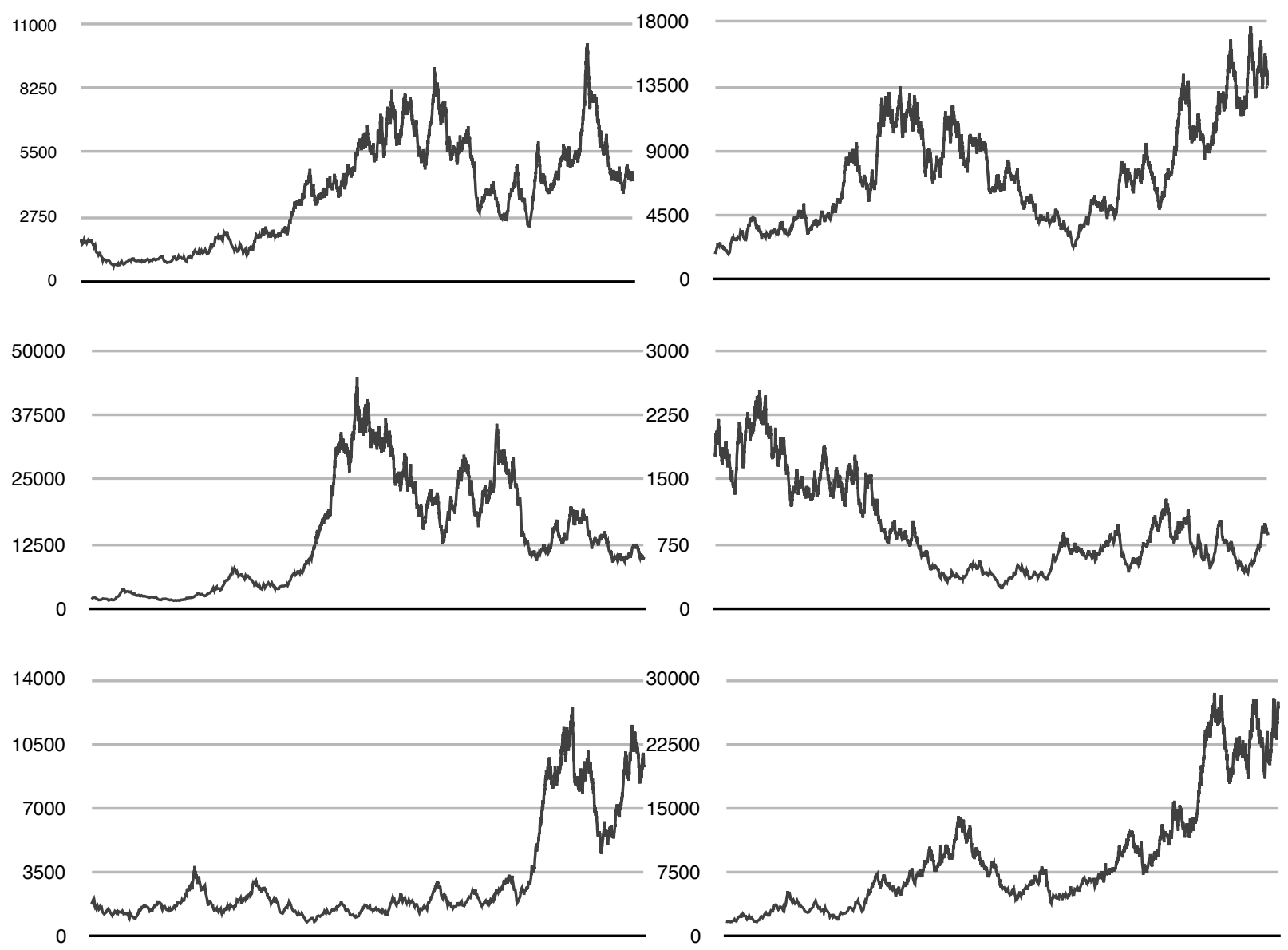

Figure 13

This figure depicts realizations of the analytical Mandelbrot Market-Model. The basis of these realizations are the DAX Performance-Index 1994 to 2013 (20 years). After taking the logarithm of the price series, we decompose the resulting series using the dyadic, recursive decomposition at scales 32,16,8, and 4. Assuming lognormal distributions, we estimate for each scale the mean and the standard deviation of the logarithm of the trend size and the logarithm of the trend slope statistics. Additionally, we estimate the two covariances between these two characteristics using a Cholesky decomposition and separated by up or downward trends. Because of the law of large numbers we model the residual (after removing all trends) as normal distributed (in this log space). The realization process is similar to the trend bootstrapping scheme. Drawing an initial sign (uniformly distributed), we alternate generating upward and downward trends per scale. Each trend is concatenated to the former one. The trends are constructed by drawing a two component vector form the normal distribution with zero mean and standard deviation of one, where the two components correspond to the trend size and the trend slope. We then multiply the upward or downward covariance matrix based on the current sign of the trends. After adding the means of the trend sizes and trend slopes, we apply the exponential function. This construction is executed for all dyadic scales 4,8,16,32 and the result is summed up. Finally, we add for each data point the residual which is modeled using a normal distribution and add the main trend (of the beginning of this analysis process). Finally, we apply the exponential function to transfer the realization to the original price space. 